\title{
«Vergine bella, che, di sol vestita»: 'fragmenta' spirituali di Petrarca nella poesia francese del Cinquecento
}

\section{Alessandra Preda}

\section{(2) OpenEdition}

Journals

\section{Edizione digitale}

URL: https://journals.openedition.org/studifrancesi/40146

DOI: $10.4000 /$ studifrancesi.40146

ISSN: 2421-5856

\section{Editore}

Rosenberg \& Sellier

\section{Edizione cartacea}

Data di pubblicazione: 1 juillet 2004

Paginazione: 73-94

ISSN: 0039-2944

\section{Notizia bibliografica digitale}

Alessandra Preda, ««Vergine bella, che, di sol vestita»: 'fragmenta' spirituali di Petrarca nella poesia francese del Cinquecento», Studi Francesi [Online], 142 (XLVIII | I) | 2004, online dal 30 novembre 2015, consultato il 09 septembre 2021. URL: http://journals.openedition.org/studifrancesi/40146 ; DOI: https://doi.org/10.4000/studifrancesi.40146

\section{(c)}

Studi Francesi è distribuita con Licenza Creative Commons Attribuzione - Non commerciale - Non opere derivate 4.0 Internazionale. 


\title{
DISCUSSIONI E COMUNICAZIONI
}

\section{«Vergine bella, che, di sol vestita»: 'fragmenta' spirituali di Petrarca nella poesia francese del Cinquecento}

\author{
«Perché cantando il duol si disacerba» \\ (F. Petrarca, Rerum Vulgarium \\ Fragmenta, «Canzone 23», v. 4)
}

Il prestigio di Petrarca nelle lettere francesi risale all'epoca stessa del grande umanista: fra il Tre e il Quattrocento l'autore del De remediis gode Oltralpe di un grande successo, oggetto di imitazione quanto di polemica nel dibattito che anima la cultura francese e il suo tentativo di affermazione nazionalistica ${ }^{1}$. Alla diffusione e all'influenza della produzione latina che guadagna al Petrarca l'appellativo di «devotissimus catholicus ac celeberrimus philosophus moralis» ${ }^{2}$ si accompagna, alla fine del Quattrocento, l'interesse per i Trionf. L'impianto allegorico di quest'opera in volgare, la preoccupazione morale e pedagogica che ne alimentano la sostanza, rinnovano l'ammirazione per il Petrarca presso un pubblico educato dalla tradizione del Roman de la Rose. Così, nel Cinquecento, dopo la riduzione in prosa di Georges de La Forge (1514) si pubblica la traduzione in versi di Simon Bourgouyn (1530) che premette ad ogni Trionfo un breve commento storico-morale, anticipando la cura con cui, nel volgarizzamento di Jean Meynier (1538), gli insegnamenti del Petrarca verranno parafrasati da brevi versi esplicativi.

Negli stessi ambienti in cui risplendono i preziosi manoscritti dei Trionfi-e si cita il celebre esemplare di Anne Louis Malet de Graville ${ }^{3}$ - compaiono le prime copie

(1) F. Simone, Rinascimento francese. Studi e ricerche, Torino, SEI, 1961, pp. 141-222. La bibliografia sulla fortuna francese del Petrarca, ancor prima del petrarchismo poetico, è immensa. Ci limitiamo a segnalare importanti contributi di sintesi: $\mathrm{N}$. Mann, La fortune de Pétrarque en France: recherche sur le "De remediis", «Studi Francesi», 37, 1969 pp. 1-15; Id., Petrarch's role as moralist in fifteenthcentury France, in A.A. Humanism in France at the end of the Middle Ages and the early Renaissance, ed. by A.H.T. Levy, Manchester-New York, Manchester U.P., Barnes \& Nobles Inc., 1970, pp. 6-28; D. Cecchetti, Il Petrarchismo in Francia, Torino, Giappichelli, 1970; E. Balmas, Prime traduzioni del Canzoniere nel Cinquecento francese, in «Saggi e studi sul Rinascimento francese», Padova, Liviana, 1982, pp. 3-21; J. Balsamo, Le 'Pétrarque François'. La constitution d'un mythe littéraire et son rôle pour les poètes français du XVI ${ }^{e}$ siècle, in AA.VV., La littérature et ses avatars: discrédits, déformations et rébabilitations dans l'bistoire de la littérature, Paris,
Aux Amateurs de Livres, 1991, pp. 89-97; D. CECChetti, Petrarca in Francia prima del Petrarchismo: un mito polemico, «Franco-Italica», 1997, pp. 7-31;

E. Duperray, L'or des mots: une lecture de Pétrarque et du mythe littéraire de Vaucluse des origines à l'orée du XX $X^{e}$ siècle. Histoire du pétrarquisme en France, Paris, Publications de la Sorbonne, 1997; AA.VV., Dynamique d'une expansion culturelle. Pétrarque en Europe du XIV au XX $X^{e}$ siècle, Paris, Champion, in corso di stampa.

(2) J. De MontreuIL Opera: 1. Epistolario, ed. critica a cura di E. Ornato, Torino, Giappichelli, 1963, p. 208 e Devotissimi catholici, p. 315. Cfr. D. CECCHETTI, Temi umanistici nell'opera di Jean de Montreuil, «Le Moyen Français» 8-9, 1981, pp. 37-110.

(3) E. Pellegrin, Manuscrits de Pétrarque dans les bibliothèques de France, Padova, Antenore, 1966. III, p. 472. Il manoscritto è conservato presso la Sala Manoscritti della Bibliothèque Nationale di Parigi, (ms. Franç. 22541, La Vallière 6). 
dei sonetti del Petrarca ${ }^{4}$, a riprova del ruolo di transizione che l'opera allegorica riveste nella diffusione del "mito petrarchesco", tra gli scripta latini e le rime sparse del Canzoniere. Rime sparse, appunto, quelle che circolano tra gli epigoni del XV secolo in terra francese, ma impreziosite da un'aura di squisita spiritualità che si conviene al probatus auctor, ma soprattutto al sanctus Petrarcha del De remediis. Segnatamente, fra questi fragmenta, si afferma l'ultimo componimento della raccolta lirica, la Canzone 366, «Vergine bella, che, di sol vestita». Tradotta in esametri latini da Filippo Beroaldo, il Canticum de laudibus divae Virginis conosce un primo successo francese nelle diverse edizioni lionesi e parigine dell'opera del noto umanista ${ }^{5}$. Il timbro tradizionale della supplicatio predomina nel Canticum di Beroaldo, una versione fedele che tende a sfumare, tuttavia, il tratto concreto - e spesso ossimorico - della Canzone petrarchesca:

\author{
vergine bella \\ virginal claustro, \\ altissima humilitate \\ Con le ginocchia della mente inchine \\ prego che sia mia scorta \\ Mortal bellezza, atti et parole m'anno \\ tutta ingombrata l'alma
}

\author{
virgo decens \\ sacratissima claustra \\ Eximia [...] mansuetudine \\ Ad te confugio supplex: et supplice corde \\ Sis mihi præsidium quæso [...] \\ Femineum decus et mores mellitaque verba \\ Turbarunt mentem $[\ldots]^{6}$
}

Il successo di questa versione latina della Canzone è alimentato dal commento esplicativo con cui Josse Bade, nel 1507, arricchisce l'edizione dell'opera di Beroaldo. Bade si è già avvicinato all'opera latina di Petrarca editando e commentando il Bucolicum carmen ${ }^{7}$ : come lui stesso ribadisce nella prefazione alla sua edizione parigina ${ }^{8}$, la profonda preoccupazione spirituale che anima le egloghe e ne nutre il tessuto classico rende il testo di Petrarca interessante e legittima il suo commento esplicativo. Anche dove il Petrarca racconta l'amore per Laura e cita il ramo del lauro dato a Stupeus per impietosire Dafne, l'umanista commenta:

Subtilis fictio: intelligo enim illum aureum ramum quem Æneas in Virgilio petiit, id est prudentiam seu sapientiam sine qua nemo in pœtarum recipitur cœtum ${ }^{9}$

Ugualmente, l'ampia digressione erudita sul termine peana, che dà il titolo alla versione latina della Canzone alla Vergine, ricolloca il poema in un contesto umanisti-

(4) Alcuni sonetti del Petrarca sono stati copiati in un manoscritto ora presso il museo Dahlem di Berlino, compilato da Louis Malet de Graville. Tali sonetti sono stati segnalati nella tesi di M.K. WHITE, Pétrarque et le Pétrarquisme en France avant la Pléiade (Liverpool, 1972). Cfr. G. Mombello, I manoscritti delle opere di Dante, Petrarca e Boccaccio nelle principali librerie francesi del secolo XV, in AA.VV., Il Boccaccio nella cultura francese, a cura di C. Pellegrini, Firenze, Olschki, 1971, pp. 81-209.

(5) La traduzione latina conosce una prima edizione nella seconda parte del volume Orationes Philippi Beroaldi viri clarissimi Bononize littaras bonas docentis, Lyon, J. Trechsel, 1492. Essa viene poi rieditata con il commento di Josse Bade nel 1507, Paeanes divae Virginis ex Francisci Petrarcae poemata vernaculo in latinum conversi a Philippo Beroaldo, Impressum est rursus Parrbisiis opera Johannis Barbier, XI Kalendas martias MDVI. Due anni dopo viene inserita all'interno di un'altra raccolta di versi di FILIPPO BEROALDO, Carmen lugubre Philippi Beroaldi de dominicae Passionis die.
Eiusdem paenes sive canticum de laudibus divae Virginis (ex Francisci Petrarchae poemate vernaculo in latinum conversi). Ab Ascensio dilucide explanata, Impressum Parisiis in Bellovisu, pro Johanne Petit, anno Domini MDIX, In- $4^{\circ}$.

(6) Carmen lugubre Philippi Beroaldi de dominicae Passionis die. Eiusdem paenes sive canticum de laudibus divae Virginis (ex Francisci Petrarchae poemate vernaculo in latinum conversi). Ab Ascensio dilucide explanata, Impressum Parisiis in Bellovisu, pro Johanne Petit, anno Domini MDIX, esemplare della Bibliothèque Nationale di Parigi, Rés. p. Z. 69 (8), ff. $\mathrm{Xv}^{\circ}$ vv. 57-57, f. XIr ${ }^{\circ}$ vv. 76-77.

(7) Francisci Petrarchae potae insignis Bucolica Carmina in duodecim oeglogas distincta et diligenter ab Jodoco Badio Ascensio explanata, Venundantur Parrhisiis in regione divi Jacobi sub leone argenteo et pelicano, 1502, esemplare della Bibliothèque Nationale di Parigi, Rés. M. Yc, 250. 175.

(8) Cfr. F. Simone, Il Rinascimento ...cit, pp.172

(9) Francisci Petrarcae, cit., f. IXr ${ }^{\circ}$. 
co e rievoca i nomi di Pindaro, Virgilio e Stazio a testimonianza di una familiarità con i classici che caratterizza Beroaldo quanto il poeta «tuscus» dell'antica «ethruria» ${ }^{10}$.

Virgo decens. Hoc carmen primum vernacula italorum lingua a Francisco Petrarcha compositum et deinde a Philippo Beroaldo in latinam productum inscribit peanes. quo titulo inscribi solent apollinis laudes [...]. Juvenalis saty.vi. Parce precor pean: et tu depone sagittas. Sulpitius vult peana opus pindari de laudibus apollinis neutro genere et numero tamtum plurali enunciari: verum Vergilius lib. vi. dicens laetum choro peana canentes. Et Statius dicens Herculeum peana canunt $[. . .]^{11}$

$\mathrm{Al}$ «religiosissimo» destinatario, Beroaldo loda anzitutto le qualità cristiane del «religioso» Petrarca

Superrime cum feriatus essem vir præstantissime periclitaturus ingenium converti in latinam sermonem ex vernacula lingua canticum Francisci Petrarche religiosum quod tibi homini religiosissimo potissimum dicavi ${ }^{12}$

La sostanza spirituale del dotto componimento riappare anche tra le righe del commento, che non si esaurisce in un corredo di osservazioni filologiche: per legittimare la traduzione dell'opera lirica e in volgare dell'inedito Petrarca, Bade pone l'incontro tra i due umanisti sotto il segno di un impegno etico e didascalico ${ }^{13}$ che rende il "peana mariano" felice espressione di una cultura classica cristiana.

La versione latina della Canzone e il commento di Bade vengono nuovamente editate nel corso della prima metà del Cinquecento, a Lione, nel $1523^{14}$ e a Provins nel $1526^{15}$. L'ultimo poema del Canzoniere si trova ad essere così il primo fragmentum delle Rime divulgato con successo Oltralpe, un tassello prezioso che, paradossalmente tradotto in latino, permette al probatus filosofo morale di introdurre il poeta d'amore.

Come è noto, Clement Marot, a sua volta, traduce un poema di Beroaldo il Carmen lugubre de dominicae Passionis die, e conosce la bella versione della preghiera alla Vergine, exemplum di un'ortodossia cattolica con cui il poeta di Cahors - in questi anni al servizio di Marguerite d'Angoulême, in contatto con gli "Evangéliques de Meaux" - preferisce non misurarsi: incline a innestare nella tradizione allegorica medievale un'inedita quanto moderna spiritualità, traduce la canzone $323^{16}$, cioè «Standomi un giorno solo a la fenestra». Si tratta del poema detto comunemente la canzone delle visioni ${ }^{17}$, perché composto da sei quadri allegorici su alcuni dei più noti

(10) Carmen ...cit., f. XIr ${ }^{\circ}$.

(11) Ibidem, f. IXr ${ }^{\circ}$.

(12) Ibidem, «Philippus Beroaldus Reverendo domino Jacobo Antiquario ducali secretario S. D.», f. VIII vo.

(13) Ibidem, f. XIr ${ }^{\circ}$

(14) Carmen [...] Impressum Lugduni, per Claudium Nourry, in-4 ${ }^{\circ}, 1523$, esemplare della Bibliothèque Nationale di Parigi, Rés. p.Yc.33.

(15) Carmen [...], Imprimé à Provins par la veuve Jeban Trumeau et Guillaume Trumeau, libraire demeurant à l'Homme saulviae, près le Pont au poysson, in- $8^{\circ}, 1526$, esemplare della Bibliothèque Nationale di Parigi, Rés. p.Y. c. 947.

(16) C. Marot, Euvres poétiques, éd. par G. Defaux, Classiques Garnier, Paris, Bordas, 1990, p. 347: «Le Chant des Visions de Petrarque, translaté de Italien en Françoys». Come è noto, in seguito, Marot traduce sei sonetti del Canzoniere, pubblicati nella prima edizione postuma della sua opera data alle stampe a Lione, nel 1544. Si tratta dei sonetti: $1 ; 161 ; 248 ; 338 ; 346 ; 348$.
(17) Cfr. F. Maggini, La canzone delle visioni, «Studi Petrarcheschi», I, 1948, pp. 37-50; FREDI ChIAPPelli, Studi sul linguaggio del Petrarca. La canzone delle visioni, Firenze, Olschki, 1971; M. FEO, Il sogno di Cerere e la morte del lauro petrarchesco, in Il Petrarca ad Arquà. Atti del Convegno di studi nel VI centenario, a cura di G. Billanovich, Padova, Antenore, 1975, pp. 117-48; F. ZAMBON, Sulla fenice del Petrarca, in Miscellanea di studi in onore di V. Branca. I, Firenze, Olschki, 1983, pp. 411-25; R. Bettarini, Postille e varianti nella canzone delle visioni, «Studi Petrarcheschi», II, 1985, pp. 159-84; P. FRARE, Dalla contrapposizione alla identificazione: l'io e Laura nella canzone delle visioni, «Strumenti critici», XV, 3, 1991, pp. 387-403; B. MarTinelli, Veduta con naufragio, RVF 323, «Italianistica», XXI, 1992, pp. 511-535; S. AGOSTI, Gli occhi, le chiome. Per una lettura psicanalitica del Canzoniere del Petrarca, Milano, Feltrinelli, 1993, pp. 67-78; M. Santagata, Il naufragio dei simboli, «Chroniques italiennes», 41, 1995, pp. 19-41. 
simboli laurani - rispettivamente una fiera, una nave, un lauro, una fenice, una donna - e distribuiti secondo una struttura a polittico. Stimmate funebri segnano ciascuna delle visioni, in cui si realizzano sei adynata - la morte, cioè, di quel che non può morire, - dall'implosione definitiva della sorgente di Valchiusa, al lauro fulminato per sempre, vero hapax dell'intero Canzoniere ${ }^{18}$. L'ultima strofa descrive la morte di Laura, evocata dal mito euridiceo, cui non pone rimedio né l'amore, né la poesia: la «bella donna» rinasce solo in una prospettiva ultraterrena, «Lieta si dipartio, nonché secura» ${ }^{19}$. L'allegorismo, accentuato dal clima idillico dell'ambientazione, perfettamente tradotto nella versione francese, l'assunto meditativo e il messaggio etico che attraversa esplicitamente questa Canzone, facilitano l'affermarsi di una produzione volgare che appare in assoluta sintonia con l'opera già nota del Poeta incoronato. Del resto, il commento con cui Petrarca viene diffuso in Francia è quello a cura di Francesco Filelfo ${ }^{20}$, incline a valorizzare la materia morale della lirica volgare petrarchesca.

Rispetto al modello, la 'belle fidèle' di Marot comporta in verità una variante di interesse: se il Petrarca stesso apprende dalle sei visioni della sua Canzone «un dolce di morir desio ${ }^{21}$ in una prospettiva spirituale, che la critica moderna ha riletto anche in termini squisitamente estetici ${ }^{22}$, Marot attribuisce al Re, François $\mathrm{I}^{\mathrm{er}}$, «ung doulx desir / De briefvement soubz la terre gesir» (vv. 75-76). Smorzato l'ossimoro petrarchesco che chiude in posizione accentuata il congedo, Marot sottolinea il valore di un riposo più metaforico, una saggezza che Francois $\mathrm{I}^{\mathrm{er}}$ detiene anche in nome della profonda frequentazione delle lettere, segnatamente del Petrarca. Nel 1533 Marot aggiunge al titolo della sua traduzione «translaté de Italien en Françoys par le commandement du Roy», sottolineando un rapporto tra la diffusione del petrarchismo e il re destinato a segnare la fortuna del Canzoniere in Francia ${ }^{23}$. Di quegli anni è l'apparente scoperta della tomba di Laura ad Avignone, simbolico crocevia delle relazioni franco-italiane che il Re visita opportunamente ${ }^{24}$. Le reliquie di Madonna Laura concretizzano in uno spazio storico geografico la translatio studii in cui il Reale Pellegrino riconosce le istanze di una novella egemonia politica francese. Ad Avignone, fra nomi e luoghi noti, si compie il percorso che traduce Petrarca in Francia e ad Avignone, appunto, Vasquin Philieul pubblica nel 1555 la prima traduzione completa del Canzoniere. Valchiusa, la Provenza, la sorgente della Sorgue acquistano in tale versione un inedito valore. Il desiderio politico-culturale di appropriarsi di un'eredità che ha radici locali ma che aspira a riconoscimenti nazionali si sposa, nell'opera di Philieul, al tentativo di riproporre il cammino metaforico del viator Petrarca: da questi luoghi a mete ben più spirituali, che sono la sostanza dell'intera impresa poetica. In tale viaggio, la palinodia

(18) Cfr. M. FEo, Il sogno di Cerere e la morte del lauro petrarchesco, in Il Petrarca ad Arquà, Padova, Antenore, 1975, pp. 117-148.

(19) F. Petrarca, Rerum Vulgarium Fragmenta, Canzone 323, v. 71; cfr. C. MAROT, op. cit., p. 349, v. 71, «Puis asseurée en liesse est saillie».

(20) Il commento del Filelfo fu redatto intorno al 1443 ed ebbe una larga circolazione manoscritta anche in terra francese, cfr. E. Pellegrin, Manuscrits de Pétrarque dans les bibliothèques de France, Padova, Antenore, 1966 , p. 145 , p. 323 et p. 350 ; G. Mazzatinti, Inventario dei mss. italiani delle Bibl. di Francia, Roma, 1886, I, p. 10. Pubblicato una trentina d'anni dopo, («impressum Bononiae, anno domini Mccclxxvi, ad instantiam et petitionem Sigismundi de' Libri») viene stampato nuovamento nel 1478 (Venezia, Teodoro di Reynsburch e Reynaldo de Novimagio) e nel 1481 (Venezia, Leonard Wild). Nel 1484 esce la fortunata edizione veneziana di Piero di Plasi con il commento integrato dello Squarzafico e, per i Trionfi, con la sposizione di Bernardino Ilicino, che costiutisce la vulgata editoriale del Petrarca commentato. Sul commento del Filelfo cf. E. RAImONDI, Francesco Filelfo interprete del Canzoniere, «Studi Petrarcheschi», 3, 1950, pp. 143-64; G. Belloni, Laura tra Petrarca e Bembo. Studi sul commento umanistico-rinascimentale, $\mathrm{Pa}$ dova, Antenore, 1992.

(21) «Canzon, tu puoi ben dire / Queste sei visioni al signor mio / An fatto un dolce di morir desio»

(22) P. Frare, art. cit.

(23) Come è noto lo stesso François I ${ }^{\mathrm{er}}$ traduce le rime del Petrarca, cfr. E. Balmas Prime traduzioni del Canzoniere nel Cinquecento francese, art. cit., pp.12-13.

(24) Cfr. O. Millet, Le tombeau de la mort et la voix du poète: la mémoire de Pétrarque en France autour de 1533 in Regards sur le passé dans l'Europe des XVI ${ }^{e-}$ XVII ${ }^{e}$ siècles, Bern, Lang, 1977. 
alla Vergine riacquista la sua naturale collocazione come ultimo componimento della lirica d'amore, epigono luminoso di un percorso che valorizza la Canzone finale ma limita - come vedremo - la fortuna del fragmentum.

Vasquin Philieul appartiene ad una famiglia del Comtat Venaissin conosciuta fin dal XIV secolo ${ }^{25}$. Romain Filhol (Philiol, Philieul, Filioli), sieur de la Magdaleine, notaire de Carpentras, fra i primi a pubblicare gli Statuta comitatus Venayssini (1511), è suo padre. Vasquin si cimenterà a tradurre gli Statuts nel 1558, fregiandosi per l'occasione di un ritratto che documenta la sua data di nascita, il $1522^{26}$. Docteur-es-droit ${ }^{27}$ tra il 1548 e il 1555, Vasquin Philieul diviene negli anni sessanta canonico di Notre Dames des Doms ${ }^{28}$ ad Avignone e giudice alla corte temporale del Comtat. Ufficialmente riconfermato in tale carica nel $1562^{29}$, il canonico frequenta gli ambienti altolocati della curia di Avignone, in particolare il milieu italianisant che circonda il potente Francesco Fabrizio de' Serbelloni, nipote di Papa Pio IV, inviato ad Avignone dallo stesso pontefice per sedare i tumulti protestanti $\mathrm{i}^{30}$. Amico di Louis de Pérussis, viguier di Avignone, Philieul offre un dizain di elogio ai suoi Discours des guerres de la Comté de Venayscin ${ }^{31}$, a testimonianza della stima per un'opera impegnata nella lotta contro il diffondersi della Riforma e per un protettore quale «Fabrice le grand $»^{32}$ cui i $D i$ scours sono dedicati. Lo stesso Philieul omaggia il Serbelloni di un sonetto in italiano ${ }^{33}$ che garantisce le sue competenze di traduttore e sancisce il suo essere partecipe, a pieno titolo, di un'operazione culturale che in quegli anni converte i successi d'Ol-

(25) Cfr. C.-F.-H. Barjavel, Dictionnaire historique, biographique et bibliographique du département de Vaucluse, Carpentras, L. Devillario, 1841, vol. II, pp. 263-267. Sulla vita di Vasquin Philieul cfr. E. PICOT, Les Français italianisants au XVIe siècle, Paris, Champion, 1907, vol. II, pp. 43-48; M. Francon, Vasquin Philieul traducteur de Pétrarque, «French Studies», 1950, pp. 216-226; G. Bellati, Il primo traduttore del Canzoniere petrarchesco nel Rinascimento francese: Vasquin Philieul, «Aevum», 1985, pp. 371-398; R. ANTONELLI, Traduzione e destrutturazione. "Rerum vulgarium fragmenta", le versioni di Clement Marot e V. Philieul, «Micromégas», XVIII-1-2, 1991, pp. 11-20.

(26) Cfr. Les Statuts de la Comté de Venaiscin, Avignon, Claude Bouquet, 1558, f. fr ${ }^{\circ}$ «Anno Ætatus suæ XXXIIII. 1556 Vasquini Filioli Effigies».

(27) Solo nel 1555, Philieul si qualifica in tal senso: Toutes les euvres vulgaires. [...] mis en Françoys par Vasquin Philieul de Carpentras, Docteur es Droictz. Nel testo del 1548, Laure d'Avignon, il titolo non compare.

(28) Bibl. di Carpentras, ms. 1361, fol. 390.

(29) J.-H. (Abbé) ABANĖs Gallia Christiana novissima. Histoire des archevêches, évêches et abbayes de France et de Prouvance [...], Avignon, 1920, t. VII, nella lista dei canonici di Avignone, Vasquin Filhol 1563, 1586.

(30) Francesco Fabrizio de' Serbelloni, nato a Milano da Elisabetta e Pietro Serbelloni (la cui sorella Cecilia è la madre del futuro pontefice Pio IV) fu inviato nell'ottobre del 1561 in Francia per difendere Avignone minacciata dagli Ugonotti, cf. Avviso di Roma del 25 ottobre 1561, Urb. 1039, p. 305, Biblioteca Vaticana. Nominato comandante generale delle truppe della Santa Sede nel Vénayssin, entrò ad Avignone il 29 novembre 1561 e ripartì per l'Italia nel gennaio del 1565 . Cfr. C.-F.H. BARJAVEL, Dictionnaire historique, biographique et bibliographique du département de Vaucluse, Carpentras, L. Devillario, 1841, vol. II.

(31) Discours des guerres de la Comté de Venayscin, et de la Provvence: ensemble quelques incidentz, le tout dedié à l'illustrissime, et excellentissime seigneur, et chevalier, monseigneur Fr. Fabrice de Serbellon, cousin germain de N.S.P. et son General en la cité d'Avignon, et le dicte Comté: par le seigneur LOYS de Perussis [...], Avignon, Pierre Roux, 1563 , verso del frontespizio, «A tres vertueux et tres noble seigneur, le S. Loys de Perussiis, Escuyer de Coumons, Vasquin Philieul S.».

(32) Ibidem, «Par toy, Loys, reluist tout Avignon. / Quoy Avignon? Mais bien la Sphere ronde, / Ou est espars de Jesus CHRIsT le nom. / Que faict il plus le Soleil en ce monde? / Ton pur sçavoir, ta naïfve faconde, / Par hault obiect d'un Fabrice le grand, / Monstrent combien Dieu nostre seul garant, / Douant de toy Avignon l'honnorable, / L'aime et cherist: et qu'en ce monde errant / Pour bien loeur convient estre louable».

(33) Ragionamenti et ultime parole, che dice l'illustrissimo principe M. Francesco di Lorena, Duca di Guisa, nel suo trapassare, Avignon, Pierre Roux, 1563, au verso du titre «Sonetto di Vasquin Philieul». Anche i Discours di Perussis sono dedicati a Fabrizio Serbelloni. La dedica è in italiano, cf. Discours... cit., pp. 3-4: «Allo illustrissimo et eccellentissimo Signore, e Cavaliere, Monsignor Fr. Frabitio di Serbelloni [...]». 
tralpe in un'occasione di arricchimento nazionale. Jean Charrier, illustre membro del Parlamento di Provenza, prestigioso traduttore dell'opera di Contarini ${ }^{34}$ e dell'Arte della Guerra di Machiavelli ${ }^{35}$, offre la pièce liminaire che conclude la prima e parziale traduzione del Canzoniere, pubblicata a Parigi da Philieul nel $1548^{36}$.

Vasquin aussi en la region mesme

Beut mesme humeur: mais puys print meilleur theme

Quand translata pour la Royne ce livre ${ }^{37}$

I versi di Charrier evocano l'idillica parentela geografica tra il poeta aretino 'provenzalizzato' e il canonico di Carpentras che si cimenta, in questa prima edizione dedicata a Caterina de' Medici, con 229 delle 366 composizioni del Canzoniere. L'incontro, all'ombra della regina francese di terra toscana, è destinato al successo: qualche anno dopo Philieul traduce l'intera opera volgare di Petrarca e in qualità di suo concittadino ideale, la pubblica presso l'editore più prestigioso di Avignone, Barthélémy Bonhomme ${ }^{38}$. Il poeta di Carpentras si rivolge direttamente a Petrarca nel celebrare le due donne "avignonesi" amate con lo stesso ardore anche se con esiti poetici differenti:

Dont de nous deux souvent ainsi je pense:

Que si ta dame estoit de noble ligne,

Pour tout certain la mienne est sa cousine.

Ta dame avoit un port grave et mignon,

Aussi pour vray elle estoit d'Avignon,

Des villes ville, et cité des cités,

Ou tes doulx chants estoient veus et vantés,

Or ma maistresse est d'Avignon aussi:

Mais mes lourdz chants n'y sont pas leus ainsi ${ }^{39}$

Il testo scelto a modello dal traduttore è l'edizione del Canzoniere curata da Alessandro Vellutello, pubblicata a Venezia nel $1525^{40}$. Come è noto il Vellutello

(34) J. ChARRIER, Des Magistratz et republique de Venise, composé par Gaspar Contarin, Paris, Galiot Du Pré, 1544.

(35) J. CHARRIER, L'Art de la guerre, composé par Nicolas Machiavellli, Paris, J. Barbé, 1546.

(36) LAURE D'A / VIGNON. / AU NOM ET AD / -veu de la Royne Catharine / De Medicis Royne de / France. I EXTRAICT DU POETE FLO- / rentin Françoys Petrarque: Et mis en / Françoys par Vasquin Philieul. I de Carpentras. I Avec privilege / du Roy, pour cinq ans. / A Paris. De l'imprimerie de Jacques Gazeau, 1548, (esemplare della Bibliothèque de l'Arsenal, $8^{\circ}$ B.L. 6449), f. $119 \mathrm{v}^{\circ}$ : «Jehan Charrier aux lecteurs. Sonnet». Charrier, che ha pubblicato la sua traduzione dell'opera di Machiavelli presso Jean Barbé, potrebbe aver messo in contatto Vasquin con l'editore parigino Gazeau. Jean Gazeau, infatti, libraio dal 1542, diventa editore nel 1548 , succedendo al cognato Jean Barbé, morto nel 1547. Cfr. Ph. Renouard, Répertoire des imprimeurs parisiens, Paris, Lettres modernes, 1965, pp. 166-167.

(37) Ibidem, vv. 9-11. Nell'edizione successiva del 1555 il sonetto (p. 409) mantiene la sua posizione finale, a chiusura dell'opera.

(38) Toutes / Les Euvres / vulgaires:de Françoys Petrarque. / Contenans quatre Livres de M. D. Laure d'A / vignon sa maistresse: jadis par luy composez en / langage Thuscan, et mis en Françoys par Vas / quin Philieul de Carpentras Docteur es Droictz. I Avecque briefz Sommaires ou Argumens requis / pour plus facile intelligence du tout. / En Avignon, Barthélémy Bonhomme, 1555 (esemplare della Bibliothèque de l'Arsenal $8^{\circ}$ B.L. 6448). L'editore lionese Mathieu Bonhomme ha iniziato l'attività ad Avignone nel 1552, affidandola al fratello Barthélémy Bonhomme, cfr. P. PANSIER, Histoire du livre et de l'impremerie à Avignon, du XIV au $X V I^{e}$ siècle, Avignon, Aubanel frères, 1922, vol. II, p. 105, «Mathieu Bonhomme, imprimeur à Lyon, avait un depôt de livres à Avignon en 1542. Dix ans plus tard, il y adjoignit un atelier d'imprimerie que dirigea son frère ». Alla morte di Barthélemy, avvenuta nel 1557, l'atelier Bonhomme era già il più importante di Avignone; dopo diverse vicessitudini verre rilevato nel 1559 da Pierre Roux.

(39) TOUTES / LES EUVRES / vulgaires, op. cit., Livre Troisieme, «Au tres honnorable Seigneur Jan Ange Papius, premier Docteur Regent en Avignon» p. 295, vv. 29-42.

(40) Le volgari opere del Petrarcha con la espositione di Alessandro Vellutello da Lucca, Stampate in Vinegia per Giovanniantonio et Fratelli da Sabbio del mese d'Agosto, l'anno del Signore 1525, (esemplare della Biblioteca nazionale di Brera, Disc. C. 388). 
non reputa originale l'ordine delle composizioni presentato nell'aldina del $1501^{41}$, e si ripropone, prima di avviare qualsiasi esposizione, di restaurare una cronologia di avvenimenti e composizioni ben più verosimile ${ }^{42}$. Per ricostruire l'esatta successione egli si avvale di tutti i dati possibili interni ed esterni, innanzitutto luoghi, cenni storici, nomi presenti nella raccolta e, soprattutto, la dettagliata biografia dei due protagonisti, Francesco e Laura. Il Vellutello, quindi, introduce la sua esposizione con i dati concreti di una storia d'amore che si è svolta in uno spazio e in un tempo precisi, come testimonia la cartina della Provenza che egli stesso allega all'edizione, accanto a brevi cenni esplicativi: Vita et costumi del Poeta ${ }^{43}$, Origine di Madonna Laura con la Descrittione di Valclusa et del luogo ove il Poeta di lei a principio si innanmorót ${ }^{44}$. Il nostro canonico di Carpentras non può che ritrovarsi in questa documentazione locale, ed accogliere la suggestione di un disegno che si concretizza nel suo stesso paesaggio d'origine.

Il Vellutello presenta la bipartizione "in vita e in morte" di Laura, secondo un calendario tradizionale che ribadisce la concretezza dell'avventura e, al tempo stesso, il suo significato universale: la storia nata il giorno della morte di Cristo è foriera della fine spirituale di Francesco; quella che segue associa alla morte fisica di Laura la possibile rinascita dal peccato dell'amante. All'interno della Storia di nascita e morte di un Dio-uomo, si innesta, altrettanto drammatica, la storia d'amore, un controcanto terreno che oppone, in alternate successioni, la fine del corpo e la nuova vita dell'anima. Ma in questo gioco di equilibrate opposizioni i Fragmenta presentano componimenti eccentrici, una sorta di appendice circoscritta dal Vellutello in un terzo agglomerato.

Saranno adunque nella prima parte posti tutti quelli, che veramente in vita di M. L. si conoscono per la loro sententia da lui essere stati scritti, Et che de suoi felici et infelici amorosi effetti et accidenti trattano, o che in quelli del suo amoroso errore mostra dolersi, o da esso errore desiderare di potersi rimuovere, o d'essersi rimosso. Nella seconda parte saranno posti tutti quelli, che dopo la morte di lei, di tal morte propriamente, o per circoscrittione, o in altra forma parlano, Et in queste due parti tutta l'opera sera contenuta. Nella terza et ultima parte fuori dell'opera, seranno posti tutti quelli, che in diversi tempi et altri soggetti, et a più persone da lui furono scritti ${ }^{45}$

Philieul rispetta fedelmente l'ordine del modello, ripresentando nell'edizione del 1555 «le premier livre» ${ }^{46}$, in vita, quello che aveva pubblicato nel 1548 come Laure d'Avignon, cui segue il secondo libro «sur la mort et trespas d'icelle» ${ }^{47}$, e il terzo, «Ou la plus part sont contenues choses aultres que d'amours, escriptes par Petrarque a divers Seigneurs et Amis» ${ }^{48}$. Un quarto libro raccoglie la traduzione dei Trion $f^{49}$. In tale ripartizione la Canzone alla Vergine torna ad avere una collocazione privilegiata alla fine della vera e propria storia d'amore che è il soggetto essenziale della raccolta del Vellutello.

(41) Le cose volgari di messer Francesco Petrarca, impresso in Vinegia nelle case d'Aldo Romano, nell'anno MDI, del mese di luglio.

(42) Le volgari opere del Petrarcha con la espositione di Alessandro Vellutello, ... cit. «TRATTATO DE L'ORDINE DE SON. ET CANZ. DEL PET. MUTATO: noi tegniamo per cosa certa, che dal poeta non ne sia stato lassato originale ordinato, ma su diversi separati fogli, e che poi l'ordine che parve di darli a colui che fu il primo a raccoglierla et metterla insieme, tutti gli altri habbiano seguitato[...] e che da noi a migliore ordine è stata ridotta», $f$. AAvii $v^{\circ}$.

(43) Ibidem, «VITA ET COSTUMI DEL POETA», ff. AAviiv ${ }^{\circ}$ BBiir ${ }^{\circ}$.
(44) Ibidem, «ORIGINE DI MAdONNA LAURA CON LA DESCRITTIONE DI VACLUSA ET DEL LUOGO OVE IL POETA DI LEI A PRINCIPIO SI INNAMORO», ff. BBiir ${ }^{\circ}-\mathrm{Ar}^{\circ}$.

(45) Ibidem, «DivisIONE DE SO.. ET CAN. DEL POE. IN TRE PARTI», f. Ar ${ }^{\circ}$.

(46) Toutes le euvres vulgaires, ...cit, «Livre premier de Laure d'Avignon», p. 9

(47) Ibidem, «Livre deuxieme de Laure d'Avignon», p. 197.

(48) Ibidem, «Livre troisieme de Laure d'Avignon», p. 293.

(49) Ibidem, «Livre quatrieme de Laure d'Avignon. Contenans les six Triomphes que Petrarque fit pour elle $[\ldots] \gg$, p. 339. 
Come è noto il commento di Francesco Filelfo non procede oltre il primo dei sonetti avignonesi (CXXXVI); a partire dall'edizione del 1484 le chiose filelfiane sono completate e accompagnate da quelle allestite da Gerolamo Squarzafico ${ }^{50}$, il quale presenta frettolosamente la Canzone 366, «posta nel fine di questi soi sonetti per dare ad intendere a tutti quanto fusse egli devoto della regina de li cieli» ${ }^{51}$. Segue la citazione dantesca, «chi non ricorre a lei sua disianza vuole volar senza ale cioè come dice Dante $>^{52}$. Vellutello, al contrario, si sofferma dettagliatamente sull'esposizione della Canzone alla Vergine: egli non possiede una solidissima cultura umanistica, ma conosce bene Dante, cioè il Landino commentatore dell'opera dantesca - commento in cui si cimenterà lui stesso ${ }^{53}$ - ed ha un'ottima conoscenza dei testi sacri, nata forse dall'assidua frequentazione della cultura lucchese ${ }^{54}$, da cui proviene il nostro letterato. La Divina Commedia e la Bibbia - antifone, salmi, profeti - sono il ricco paratesto che il Vellutello ricostruisce e commenta, una sorta di iniziazione alle infinite trame della riscrittura $\mathrm{a}^{55}$ che il traduttore, a sua volta, è destinato a rielaborare. «Nostre devotieux et bon Chrestien Petrarque» - ci informa Philieul nell'Argomento ${ }^{56}$ che precede la traduzione del poema - ha attinto dalle Sacre Scritture questa «Oraison, ou Chant, à la glorieuse Vierge Marie; ont peut lire presque toutes les louanges, que les Theologiens inspirez du Sainct Esprit ont attribuées à elle» ${ }^{57}$.

Il sintagma con cui si apre la Canzone, «Vergine bella», evoca quella via pulchritudinis che percorsero numerosi commentatori della letteratura mariana sbocciata nel XV secolo ${ }^{58}$, scrittori spirituali attenti alla bellezza fisica oltre che morale della Madonna. L'epiteto riecheggia d'altronde l'apostrofe laurana che attraversa tutto il Canzoniere, evidenziando un parallelismo di attributi che il ritmo stesso dei versi ribadisce, con i medesimi termini nella stessa sede metrica:

Vergine bella $[\ldots]$

que' belli occhi $[\ldots]$

volgi al mio dubio stato[...]

salisti al ciel onde ai miei preghi ascolti
Anima bella da quel nodo sciolta volgi a me gli occhi e i miei sospiri ascolta (Sonetto 305)

In un movimento anaforico che caratterizza il primo verso di ciascuna delle dieci stanze della Canzone, il termine Vergine sostituisce il profano nome di Laura e ne sussume le qualità più intense: questa stella e fidata guida è saggia, pura, intera, dolce, chiara, unica, come la protagonista delle rime dafnee; donna innanzitutto, destinata a uno splendore divino che esalta i tratti dell'umano. Le qualità di Laura e quindi di Maria, sono attinte spesso da una tradizione stilnovistica ${ }^{59}$ che nutre il canto d'amore

(50) Cfr. J. Allenspach- G. Frasso, Vicende, cultura e scritti di Gerolamo Squarzafico, Alessandrino, «Italia medievale e umanistica», XXIII, 1980, pp. 233-292.

(51) Rime di Francesco Petrarca, Venezia, Veronese, 1490 (esemplare della Biblioteca di Brera, AK. XIII. 19), f. Niiiiv ${ }^{\circ}$.

(52) Ibidem.

(53) La Comedia di Dante Alighieri, con la nova espositione di Alessandro Vellutello, Venezia, Per Francesco Marcolini, 1544.

(54) Cfr. G. Belloni, Un eretico nella Venezia del Bembo: Alessandro Vellutello, «Giornale storico della letteratura italiana», 157, 1980, pp. 43-74 ora in G. Belloni, Laura tra Petrarca,... cit.

(55) Cfr. G. Velli, Petrarca e Boccaccio. Tradizioni, memoria, scritture, Padova, Antenore, 1979; M. Santagata, Per moderne carte. La biblioteca in volgare di Petrarca, Bologna, Il Mulino, 1990.

(56) Vasquin Philieul antepone a gran parte delle composizioni una breve presentazione in prosa che definisce 'Argument'.

(57) Toutes ...cit., «Argument», p. 283

(58) Cfr. G. Pozzi, Petrarca, i padri della Chiesa e soprattutto la Bibbia, «Studi Petrarcheschi», 1989, pp. 125-169.

(59) Espressioni largamente topiche quali «come di questo tempestoso mare stella»(v. 67), «d'ogni fedel nocchiero fidata guida»(v. 68). «Cosa gentile» (v. 123) per qualificare la donna amata sono di indubbia provenienza stilnovistica. Cfr. F. SuITNER, Petrarca e la tradizione stilnovistica, Firenze, Olschki, 1977, in particolare cap. 5; E. WILliamSON, A Consideration of "vergina bella", «Italica», XXIX, 1952, pp. 215-228. 
anche di espressioni liturgiche, quali il claustrum virginitatis che Cavalcanti riferisce alla donna amata e che Petrarca torna a rivolgere a Maria ${ }^{60}$. L'area del profano si estende, o meglio ascende a uno spazio più rarefatto, ma non scompare là dove l'amore del Sommo Sole per la Vergine bella si annuncia come una feconda ierogamia che genera l'Incarnazione ${ }^{61}$. Nel gioco di sovrapposte memorie liriche si innesta - scarto significativo rispetto al resto del Canzoniere - il rinvio esplicito e continuo a testi biblici, ad antifone della Chiesa, all'innologia mariana. La parabola delle vergini sagge, $\mathrm{i}$ versi del Magnificat e del Salve Regina ${ }^{62}$, rieccheggiano direttamente nelle stanze della Canzone, accanto a immagini tratte con efficacia dai Padri della Chiesa, quali S. Venanzio, Sant'Agostino e Sant'Anselmo ${ }^{63}$. Gemme di devozione che il Vellutello esalta e che Vasquin Philieul non può che riconoscere, considerandole troppo esplicite e numerose. Il letterato di Carpentras, infatti, traduce fedelmente gli attributi di Maria attinti copiosamente dalle rime lauriane, contribuendo a sua volta ad arricchire una retorica d'amore raffinata quanto efficace. Un sentimento intenso evocato in antitesi alla passione, quindi tradotto in immagini di combattimento ${ }^{64}$ ma sublimato da una luminosità onnipresente e da un dinamismo verticale che struttura l'intera Canzone evocando lo spazio rarefatto dei cieli. Refrigere, port, refuge, guide ma soprattutto astre estoile Roine, Déesse, Laura risplende in Maria di un inedito bagliore - clere, luisante, celeste e coronnée - che smaterializza nell'aere l'innamoramento stesso, «Vierge sans exemplaire, / que de ta beauté clere / Fis les cieux amoureux» (vv. 97-99).

Philieul accoglie, quindi, gli epiteti di Laura che il Petrarca rianima in Maria e traduce fedelmente, per esempio, il «virginal cloistre» che il Poeta rivisita nella sua Canzone, ma dimezza le citazioni bibliche, evita il riferimento alla parabola, ignora gli epiteti con cui la Chiesa stessa tradizionalmente prega la Vergine. Affascinato da una retorica amorosa in cui convivono tratti sacri e profani, preferisce non invadere completamente zone esclusive del sacro; al contrario enfatizza i motivi più astratti della spiritualità evocata dal Petrarca, la luce ${ }^{65}$ e un generico movimento ascensionale verso quei cieli in cui la Vierge di Philieul risplende lontana, come dice lui stesso in due versi aggiunti «...là toute exaltée / En la divinité» (vv. 77-78). Philieul evita d'altronde anche i tratti più realistici della Canzone di Petrarca: non traduce la «spietata stampa» delle membra del Cristo ${ }^{66}$, gli accenni al doloroso parto ${ }^{67}$, le «ultime strida» ${ }^{68}$

(60) Cfr. la canzone alla Vergine di Petrarca (vv 76-78) e l'opera di Cavalcanti (XLIX, 5-6).

(61) C. Galimberti, Amate dal sole in Miscellanea di Studi in onore di Vittore... cit. vol. I, pp. 427-434.

(62) Cfr. «Vergine saggia, et del bel numero una / de le beate vergini prudenti» (v. 14); «Vergine santa d'ogni gratia piena» v. 40) il cui riferimento alla salutazione evangelica è diretto (Luc. I, 28).

(63) Cfr. C. Cavedoni, La canzone di Francesco Petrarca alla Beatissima Vergine, Modena, Tip. Immacolata Concezione, 1864. Per contributi più recenti alla lettura critica della Canzone 366 cfr. G. MartellotTi, Le ginocchia della mente in Scritti Petrarcheschi, Padova, Antenore, 1983, pp. 285-288; G. Gorni, Petrarca Virginis. Lettura della Canzone "Vergine bella", «Lectura Petrarce», 1987, VII, pp. 201-208; M. PETRINI, La canzone alla Vergine, «Critica Letteraria», XII, I, 82, 1994, pp. 33-42.

(64) Cfr. «Et de l'amour mondaine / Escu ferme et entier, Soubz lequel on surmonte / Monde et chair, faisant honte / A l'ennemy si fier» (vv. 32-36).

(65) Philieul elimina il riferimento diretto alla parabola, ma trattiene il motivo essenziale della luce:«Vergine saggia, et del bel numero una de le beate vergini prudenti, anzi la prima, et con più chiara lampa» (vv. 14-15); «Vierge luisante et pure / sur toute creature, / Dans qui Dieu son filz mist, / $\mathrm{Au}$ ciel toute premiere / Par la grande lumiere» (vv. 25-29). Altrove enfatizza in modo iperbolico la luminosità petrarchesca là dove anche il sole diventa «ce luisant Soleil » (v. 81). Cfr. H. NAIs, Le thème pétrarquiste du soleil dans la traduction de V. Philieul, in Mélanges Lebègue, Paris, Nizet, 1969, pp. 59-66.

(66) Vergine, que' belli occhi, che vider tristi la spietata stampa ne' dolci membri del Tuo caro figlio (vv. 43-45).

Vers moy tes beax yeux dresse, Qui virent la destresse De ton doulx et cher fils (vv. 22-24)

(67) Tu partoristi il fonte di pietate (v. 43) De pieté la source / Prend de tes flancs sa course (vv. 79-80) Del tuo parto gentil figliuola et madre (v. 50) De ton fils fille et mere (v. 28)

(68) i’ mi ritrovo sol, senza governo, et ò già da vicin l'ultime strida (vv. 70-71)

Seul je suis sans gouvert, 
di chi naufraga definitivamente. La doppia natura in cui si sostanzia Maria nella Canzone petrarchesca, tende a unificarsi, nella traduzione, in un'immagine astratta:

Vergine pura, d'ogni parte intera [...] ch'allumi questa vita, et l'altra adorni (v. 29)
Vierge parfaicte et clere [...]

Beau parament des cieux (v. 51)

Il ritmo, però, della preghiera, elogium e supplicatio in cui si risolve la Canzone, trova un'accurata corrispondenza nella versione francese: l'anaforica sequenza di una doppia aggettivazione sinonimica che qualifica la Vierge di Philieul mantiene l'euritmia dell'originale privilegiando, al piano semantico dell'intertestualità biblica, la musicalità della litania.

\author{
Vergine bella (v. 1) \\ Vergine pura (v. 27) \\ Vierge belle et ornée(v. 1) \\ Vierge luisante et pure (v. 25) \\ Vergine santa, d'ogni gratia piena (v. 40)Vierge saincte et humaine (v. 73)
}

La tradizione lirica italiana non presenta esempi di inni mariani entro un contesto profano, in posizione di tale rilievo quale l'ultimo componimento della raccolta. Petrarca si ispira genericamente alla tradizione lirica occitanica ${ }^{69}$, ma si riferisce in modo diretto a un modello estraneo alla lirica, la preghiera pronunciata da San Bernardo nell'ultimo canto del Paradiso dantesco. Il celebre incipit di questo XXXIII canto «Vergine madre, figlia del tuo figlio», in cui si ripropone l'ossimoro cristiano che verbalizza il mistero dell'Incarnazione, rieccheggia nella Canzone «Vergine pura, d'ogni parte intera, / del tuo parto gentil figliuola et madre» (vv. 27-28) ${ }^{70}$. Philieul - che elimina come abbiamo osservato il termine 'parto' - recupera l'identità etimologica e la consonanza fils / fille $e^{71}$, attingendo da una tradizione provenzale nota anche all'autore della Comedia. In un verso successivo, «madre, figliuola et sposa» (v. 47), definito come «il rovesciamento femminile dello stilema della derelitta [...] ad indicare la somma degli affetti possibili $\gg^{72}$, lo stesso Petrarca allude a un'espressione diffusa nella lirica dei tardi trovatori, da Peire Cardenal a Peire de Corbiac ${ }^{73}$. La traduzione di Philieul, «Espouse, fille et Mere» (v. 86), ripropone i termini anche se nell'ordine rovesciato, lo stesso in cui si ritrovano, per esempio in Peire de Corbiac, «Domna, espoz, filh' e maire» ${ }^{74}$. Del resto tutta la Canzone risente di suggestioni provenzaliz-

Or le dernier passage

Au celeste heritage

Me soit seur et ouvert

(vv. 129-132)

(69) Il canzoniere di Guiraut Riquier, per esempio, è suggellato da una preghiera alla vergine. Cfr. E. AuERBACH, La preghiera di Dante alla Vergine ed antecedenti elogi, in Studi su Dante, Milano, 1971, 263-291; V. Bertolucci Pizzorusso, Il canzoniere di un trovatore: il 'libro' di Guiraut Riquier, «Medioevo romanzo», V, 1978 , pp. 216-57, ora in Morfologie del testo medievale, Bologna, Il Mulino, 1989 , pp. 87-124; A. Di RosA, Le laude nella cultura poetica medievale. Note per una ricerca, in Miscellanea di studi in onore di Vittore Branca ... cit., pp. 449-58.

(70) Cfr. G. Rabuse, Petrarcas Marienkanzone im Lichteder "Santa Orazione"Dantes in Petrarca 13041374. Bertrage zu Werk und Wirkung, a cura di F. Scalk, Frankfurt, Klostermann, 1975, pp. 242-254; G. Velli, Il Dante di Francesco Petrarca, «Studi petrarcheschi», n.s., 1985, pp. 185-199; P. BOITANI
Il tragico e il sublime nella letteratura medievale, Bologna, Il Mulino, 1992, pp. 278-288; G. BARBERI SquAROTTI, La preghiera alla Vergine: Dante e Petrarca, «Filologia e Critica», XX, fasc. II-III, 1995, pp. 365-374.

(71) «Vierge parfaicte et clere, De ton fils fille et mere» (vv. 49-50).

(72) R. BETTARINI, Lo stilema della derelitta, «Filologia italiana», 41, 1983, pp. 5-16.

(73) F. Goldin, The Law'a Homage to Grace. Peire Cardenal's Vera Vergena, «Romance Philology», 20, 66-67, pp. 466-477; H.P.J.M. AHSMAnN, Le culte de la sainte Vierge et la Littérature profane du Moyen Age, Utrecht-Paris, 1930. Cfr. Anche G. Mombello, Quatre poèmes à la Vierge de Giovan Giorgio Alione in «L"Analisi linguistica e letteraria», VIII, 2000, pp. 65-106.

(74) E la canzone Domna, dels angels regina [...], vv. 57-59, in Chrestomathie provençale, di Karl BARTSCH, sesta edizione curata da E. Koscwitz, Marburg, 1904, cool. 231-234. 
zanti, evidenti soprattutto nel tratto raffinato di alcune scelte metriche ${ }^{75}$ : la rima al mezzo per esempio dell'ultimo verso delle stanze, il commiato di struttura identica alla sirma, la ricercatezza di rime paronomastiche e derivative che impreziosiscono la Canzone di un'antica doratura. Philieul sembra accogliere questo squisito accento arcaizzante che ben si armonizza al suo progetto di riappropiazione geografico-culturale dell'eredità petrarchesca: quindi ripropone puntulmente moduli frequenti della lirica provenzale e al tempo stesso struttura la sua versione secondo formule metriche inedite nel resto della raccolta, forse nel tentativo di rendere la desueta preziosità dell'originale. Al décasyllabe utilizzato quasi esclusivamente nelle traduzioni degli altri «chants» egli preferisce per l'ultimo componimento delle sestine di sixains, un verso molto breve e raro che rende impossibile la traduzione letterale - si ottengono 42 stanze dalle 10 di Petrarca - e sacrifica l'alternanza di endecasilllabi e settenari, tipica della canzone petrarchesca. Un verso breve che tradisce l'ampio e solenne movimento dell'originale, e che attinge ad una tradizione più recente rispetto alla lirica provenzale, quella dei rhétoriqueurs ${ }^{76}$ cui guarda spesso il nostro poeta di Carpentras. Tuttavia egli non riprende la raffinata leziosità con cui la Grande Rhétorique ha reso omaggio alla Vierge «Que porteresse, en portant sa portee / Ne peult porter plus precieux portaige» ${ }^{77}$. La scelta del sixain rappresenta piuttosto il tentativo, pur velleitario, di riproporre il gioco interno di quinari che Petrarca, ben più felicemente, mantiene con la rima al mezzo dell'ultimo verso strofico.

La struttura del componimento risulta troppo semplice e al tempo stesso complessa per il traduttore di Carpentras; egli ne ripropone però l'esatta collocazione all'interno della macrostruttura dell'intero Canzoniere, cogliendo un suggerimento interpretativo di Vellutello. Ė noto, infatti, che se da sempre il Petrarca ha posto la Canzone a chiusura della raccolta, solo nella redazione finale ha collocato prima dell'inno mariano un manipolo di sonetti penitenziali, dal 362 al $365^{78}$. Così commenta il Vellutello:

Essendo il Poeta delle sue colpe dolente, Et per quelle emendare, havendo tutto l'animo a pregare Dio per la sua salute volto, come habbiamo ne tre precedenti sonetti veduto $[. . .]^{79}$

Si ribadisce l'esistenza di un contesto preciso, entro il quale la Canzone alla Vergine acquista il valore di un ultimo tassello, compimento pieno di un percorso penitenziale evocato fin dal primo sonetto, considerato da Vellutello,

non primo ma ultimo Son. scritto da lui, et in escusatione del suo amoroso errore [...] venuto poi ne gli anni maturi, e del suo amoroso errore, come vedremo in fine dell'opera, riconosciuto et del tutto pentito ${ }^{80}$

Daltronde, - i commentatori lo sottolineano - l'error mio, il pianto e l'insania evocati nella preghiera finale rimandano esplicitamente al pianto vaneggiante del

(75) Cfr. W. T. ElwerT, La varietà metrica e tematica delle Canzoni del Petrarca in funzione della loro distribuzione nel Canzoniere, in Miscellanea di Studi in onore di Vittore Branca,... cit. , vol. I, pp. 389-409; M. Perugi, Petrarca provenzale, «Quaderni petrarcheschi», VII, 1990, pp. 109-181.

(76) Lo schema del sixain AABCCB risale a G. Chastellain, cfr. H. CHATELAIn, Recherches sur le vers français au XV siècle, Paris, Champion, 1908, p. 121-122. Sulle forme metriche della traduzione di Vasquin Philieul cfr. G. Bellati, art. cit..

(77) J. Molinet, Faictz et dictz, ed. N. Dupire, Paris, Satf, 1937, vol. 2, XV, «Oraison à la Vierge Marie», v. 164. Cfr. anche i vv. 178-79: «Scintille- ment compassee au compas / Du compaisseur, qui tout poise et compasse». Su tale produzione cfr. F. Rigolot Poétique et onomastique, Genève, Droz, 1977, pp. 35-38; F. CoRnillat 'Or ne mens' Couleurs de l'Eloge et du Blâme chez les Grands Rbétoriqueurs, Paris, Champion, 1994, deuxième partie, ch. IV, «La rhétorique sans tâche», pp. 483-561.

(78) Cfr. M. Santagata, I frammenti dell'anima. Storia e racconto del Canzoniere di Petrarca, Bologna, Il Mulino, 1990.

(79) Le volgari opere del Petrarcha con la espositione di Alessandro Vellutello, ... cit. , f. Xviiv .

(80) Ibidem, f. Aivo. 
sonetto proemiale, e rispondono a consapevoli intenti strutturali evidenti soprattutto nel congedo della Canzone ${ }^{81}$ : il poeta suggella con l'evocazione di Cristo il libro che si era aperto con il ricordo della sua passione. Le diverse composizioni appaiono allora momenti precisi di un percorso biografico sentimentale riletto come un itinerario dello spirito: dal peccato alla redenzione, fino alla liturgica invocazione alla Vergine, che legittima tutto il Canzoniere. Come ricorda Philieul nella dedica iniziale,

Icy voit on les passions, que l'ame

Endure et sent soubz l'amoureuse flamme:

Et pour du tout ne la laisser mourir.

Icy s'apprend qu'à Dieu fault recourir ${ }^{82}$

Le vane speranze e il van dolore si risolvono in questa Canzone 366, ribadendo quella visione agostiniana che attraversa tutta l'opera di Petrarca, in riferimento all'Agostino del Secretum, per lo meno, se non al vescovo di Ippona ${ }^{83}$. L'impostazione didattica del Secretum, in cui il riconoscimento della verità si presenta come un'acquisizione dialettica e progressiva, si trasforma, nel libro di rime, in una dimensione narrativa dal doppio intreccio, la storia d'amore e la liberazione interiore. Questo il piano psicologico e morale che il traduttore francese rispetta dettagliatamente nella sua versione: il fragmentum consacrato alla Vergine, ben incastonato nella successione "penitenziale" delle rime, illumina di un'inedita gravità l'opera intera. Se nella dedica a Caterina, che introduce il primo libro, domina il tema della translatio studii et imperi ${ }^{84}$, che caratterizza la storia - dall'Italia alla Francia-, nella dedica a Marguerite $^{85}$ che apre questo secondo libro, grava il senso della morte, la scomparsa di François $\mathrm{I}^{\mathrm{er}}$ innanzitutto, e i temi della moralità del sapere, della virtù dell'anima à lire lungo il Canzoniere,

Tresmal duisant ce livre semblera

Traictant de dueil ou n'y a que liesse:

Mais bien duisant, $\mathrm{O}$ divine Princesse,

A ta hauteur chacun le jugera

Pource qu'en toy icy l'on trouvera

Pour Laure morte une vive Deesse:

Et qui lira Vertu, Scavoir, Noblesse,

Soudainement de toy il pensera (vv. 1-8)

Al commento storico-biografico che recupera le radici locali di Petrarca, e lo rende un familier della cultura francese, si accompagna un'interpretazione spirituale della vicenda d'amore che è la vera protagonista del Canzoniere di Vellutello e della sua versione francese: il lessico dell'innamorato si arricchisce delle sfumature psicologiche ed intimistiche di un processo di ravvedimento che "smaterializza" Laura in Maria e rende la conversione del soggetto protagonista privilegiata della preghiera alla Vergine.

(81) Cfr. F. Rico, Pròlogos al Canzoniere, «Annali della Scuola Normale Superiore di Pisa», s. III, XVIII, 1988, pp. 1071-1104.

(82) Toutes ... cit., « A tres illustre et Sovveraine Dame, Madame Catherine de Medicis, Royne de France», f. 6v .

(83) Cfr. R. Caputo, Cogitans fingo. Petrarca tra «Secretum»e «Canzoniere», Roma, Bulzoni, 1987.

(84) Laure d'Avignon, ... cit., ff. $2 \mathrm{r}^{\circ}-4 \mathrm{v}^{\circ}$ : «A tres illustre et souveraine dame, ma dame Catharine de Medici». Cfr. per esempio i vv. 27-42: «O vous François, francz et divins espritz, / Pensez vous point, en lisant tant d'escritz, / Que cela soit pro- nostication, / Signifiant des cieux l'intention, / De voir ainsi par un clement destin / Joinct au Francois l'ancien Florentin? / Que si chacun d'iceux faict que tout tremble, / Que feront ilz les deux unis ensemble? / Les deux, qui ont d'heureuse renommée / Asie, Europe et Afrique semée. / Car qui voudra droictement estimer comment la France ha d'une en autre mer / Toute l'Europe en brief temps et espace / Mise en sa Main (dont sera l'outrepasse / Henry deuxiesme) et les dons precieux..».

(85) Toutes les euvres ... cit., p. 198, «A mad. Marguerite de France, fleur des Princesses, Perle d'honneur et de vertu, Sœur du Roy, etc.». 
Tale rilettura si colloca in un contesto dichiaratamente cattolico - secondo un impegno pedagogico che il traduttore testimonia anche in opere successive ${ }^{86}-\mathrm{e}$ nazionale: dall'omaggio a Caterina, che introduce la traduzione, a quello dell'ultimo libro, dedicato a Henri $\mathrm{II}^{87}$, Philieul dimostra la volontà di accedere agli onori della grande littérature ${ }^{88}$ che si impone in questi anni alla corte dei Valois. Egli offre la prima traduzione completa delle rime che "sparse", si sono ormai affermate come modello privilegiato della lirica d'amore ${ }^{89}$; egli ne traccia un percorso razionale e ne legittima la moralità; non solo, traduttore fedele e versificatore esperto, rende il suo Canzoniere un laboratorio metrico e illustra le molteplici possibilità del verso francese, a discapito di forme e misure italiane. Tuttavia, letto dai protagonisti della rinascita culturale delle lettere francesi quali Peletier Du Mans, Pontus de Tyard e Du Bellay ${ }^{90}$, egli conosce ben presto l'oscurità dell'oblio: il momento della traduzione appartiene al passato in un tempo consacrato ormai all'emulazione, quindi alla rielaborazione di cui sono protagonisti i poeti della Pléiade. Non è solo il traduttore, comunque, a cedere il passo: l'interprete del cammino petrarchesco, attento a seguire le orme più familiari del "concittadino" quanto quelle metaforiche di un itinerarium salvationis, non è destinato al successo. Lo stesso Du Bellay, che ripercorre i passi di un intero Canzoniere, non traccia un cammino rigorosamente penitenziale, ma nel suo libro di rime d'amore esprime, al più, una generica e sfaccettata aspirazione neoplatonica una «lente et sereine remontée des âmes vers le Bien ${ }^{91}$ che si allontana dall'inquietudine agostiniana, dal dramma della caduta e del riscatto ${ }^{92}$, posto sotto il segno ortodosso della Vergine. La visione quasi agiografica, insita nell'originale curato da Vellutello, rende l'ammirazione per la poesia petrarchesca una conseguenza là dove è il lauro innanzitutto a generare l'imitazione, quelle sacre fronde in grado di sublimare la frustazione amorosa in tensione poetica.

L'errore, più che nella sovversione dei valori cristiani, consiste nel prevalere di istanze irrazionali, nell'instabilità psicologica e affettiva che è nella frammentarietà del titolo, epitome del protagonista ${ }^{93}$ : la 'conversione' si manifesta come riconquista dell'autocontrollo, della distanza nata dalla scrittura; la valenza morale si sostanzia nell'operazione letteraria che è il libro in quanto tale, a prescindere dalla storia che racconta, definitiva silloge, unità di frammenti sparsi, dalle nugæz al monumentum. Tale agli occhi di Ronsard in primis la coscienza stilistica di cui appropriarsi, invocan-

(86) Philieul traduce il De frequenti usu sanctissimi Eucharistiae sacramenti libellus (Traité de souvent recevoir le Saint Sacrement de l'Eucharistie), opera del padre gesuita Cristobal Sanchez di Madrid, in collaborazione con altri teologi del suo Ordine, pubblicata dapprima a Napoli, nel 1556 ed ampliata in un'edizione successiva a Roma, nel 1557. La traduzione di Vasquin Philieul appare ad Avignone nel 1566. Cfr. J.-F. GiLmont, Les écrits spirituels des premiers jésuites, Roma, Institutum historicum Societatis Iesu, 1961, in particolare pp. 256-259; V. DENTE, Il primo libro di un gesuita sulla Comunione frequente, «La Civiltà Cattolica», 1933, III, pp. 453-465, pp. 569-577-IV, pp. 258-71.

(87) Toutes les euvres ... cit., p. 340: «Au tres chrestien Roy Henry deuxieme».

(88) Nel 1559 Philieul pubblica a Parigi la sua traduzione dell'opera di Girolamo VIDA, Scacchia Ludus (Le jeu des Eschecz) e nel 1561 dà alle stampe lionesi di Guillaume Roville la sua traduzione del famoso Dialogo delle imprese militari e amorose (Dialogue des devises d'armes et d'amours) di Paolo Giovio. La traduzione dell'opera del gesuita spagnolo, pubblicata ad Avignone, nel 1566, riveduta e corretta viene rieditata con successo a Parigi nel 1581, con il titolo Le Traité de la fréquente communion.

(89) Dopo Clément Marot, Jacques Peletier du Mans pubblica nelle sue Cuvres poétiques (1547, Paris, Vascosan, Galiot du Pré et Corrozet) la traduzione di dodici sonetti di Petrarca. Con il 1549 inizia la pubblicazione dei Canzonieri di autori francesi da Du Bellay, Tyard, a Ronsard, Magny e molti altri, che attingono copiosamente alle rime del Petrarca e dei suoi imitatori italiani del Quattro e Cinquecento.

(90) J. Du Bellay, La Deffence et Illustration de la langue francoise, Paris, F. Morel, 1561, f. $8 \mathrm{v}^{\circ}$. Si cita la traduzione di Philieul senza nominarne l'autore. Cfr. J. Balsamo, Les rencontres des Muses, Genève, Editions Slatkine, 1992, p. 223.

(91) J. Du Bellay CEuvres poétiques, édition D. Aris et F. Joukovsky, Paris, Bordas, Classiques Garnier, 1993, tome I, «Introduction», p. XXI.

(92) A. GENDRE, Vade-mecum sur le pétrarquisme français, «Versants», 7, 1985, p. 53.

(93) Cfr. F. Rico, Rime sparse, Rerum vulgarium fragmenta. Para el titulo y el primer soneto del Canzoniere, «Medioevo Romanzo», III, 1976, pp. 101-138. 
do le Muse o il libro stesso, alla ricerca di una forma in cui si risolva ogni accidente. E a tale ricerca, in verità, si rivolge lo stesso Petrarca nella sua invocazione mariana chiedendo un sostegno, per «resurgere» cioè purgare «e' ngegno et stile, / la lingua e'l cor»(vv. 127-128). La Vergine della sua Canzone è anche tale forma perfetta, coincidentia oppositorum, segno finale di una scrittura che vanifica le distanze, permette equilibri ossimorici, riunisce infiniti generi e tradizioni nell'affermazione di una pace, che è innanzitutto armonia ${ }^{94}$. Valga di esempio il commiato della preghiera petrarchesca:

Il dì s'appressa, et non pote esser lunge,

sì corre il tempo et vola,

Vergine unica et sola,

e'l cor or conscientia or morte punge.

Raccomandami al tuo Figliuol, verace

homo et verace Dio,

ch'accolga 'l mio spirto ultimo in pace (vv. 131-137)

La Canzone si chiude su questa sorta di icona ritmica della ricongiunzione: il ripetersi del modulo bianario scandito da et / e di cinque versi è alimentato dalla gradazione semantica dei termini congiunti, quasi sinonimici, quali corre e vola, unica e sola fino all'inedito sinonimo di bomo e Dio che, attraverso Maria, qualifica il «tuo Figliuol». La coincidenza è ribadita dalla ripetizione dell'aggettivo verace in cui risuona la Vergine stessa e dall'enjambement che ravvicina i termini anche nella dispositio metrica. L'accento della rima al mezzo elimina quella distanza tra Dio e il soggetto che attraversa la canzone e per la quale il poeta si è rivolto a Maria: il dissidio trova pace, appunto, nell'armonia che la cetra offre al vivere. Ma è tale dissidio a essere negato nella versione di Philieul: la sua Vierge dimora in cieli limpidi e lontani, a discapito del gioco di riavvicinamento e distanze cui dà forma la Canzone del Petrarca.

che 'n Te Sua luce ascose (v. 3)

ben ch'i' sia terra, et Tu del ciel regina (v. 13)
Se mist dans ton cercueil (v. 6)

Combien que je sois terre,

Toy Royne d'amitié, (vv. 23-24)

Philieul sfuma gli ossimori ${ }^{95}$, allontana i termini la cui contiguità figura il paradosso dell'Incarnazione ${ }^{96}$, dirada la presenza del corpo, della terra, incapace di trasfigurare nell'arte le dissonanze del vivere. La sua è la Canzone finale, priva di senso ormai come frammento, ma perfetta come epigono di luce e purezza, che si conclude significativamente «es cieulx»:

Ainsi vole le temps:

$\mathrm{O}$ vierge mere unique

Doubt de mort me pique,

Si a moy tu n'entens.

Recommander te plaise

(94) Cfr. G. Contini, Varianti e altra linguistica. Una raccolta di saggi, Torino, Einaudi, 1970; E. BIGI, Poesia latina e volgare nel Rinascimento italiano, Napoli, Morano, 1989.

(95) Cfr. «che per vera et altissima humiltate salisti al Ciel (vv. 41-42) «Qui par Humilité / Es haults cieux est montée» (vv. 75-76).
(96) Cfr. «al vero Dio sacrato et vivo tempio fecero in Tua virginità feconda» (v. 58)

«...En ta verginité

A dieu furent un temple

Vivant, et sainct exemple

De grand fecondité» (vv. 105-108). 
Ma vie, ains mon mal aise

A ton fils precieux,

Qui mon esprit accueille

A la fin et le vueille

En paix loger es cieulx.

Il Canzoniere del Petrarca, in Italia, è sottoposto a una riscrittura cristianizzante fin dal 1536, anno della pubblicazione, a Venezia, del Petrarca spirituale ${ }^{97}$ curato dal frate minore Girolamo Malipiero. L'opera, che conosce ben sette edizioni nella seconda metà del sedicesimo secolo ${ }^{98}$, si presenta come una rielaborazione dei contenuti amorosi del testo, in grado di trasformare la donna in Madonna e la passione in Amore divino ${ }^{99}$. Alla lettura orizzontale del Vellutello, che lascia inalterato il verso ma riorganizza l'ordine delle composizioni, il Malipiero sostituisce una lettura verticale, che rispetta la sequenza dei componimenti e il loro incipit, ma altera il senso di singoli versi ${ }^{100}$. In tale contesto la Canzone alla Vergine, che non subisce alcuna alterazione, acquista un valore esemplare, in piena sintonia con il corredo di riflessioni esposte da Malipiero nell' «Ammonitione [...] alle ornate Canzoni del suo theologo et spirituale Petrarcha» ${ }^{101}$ : la pericolosa fascinazione che la poesia esercita deve plasmarsi in un canto che ecciti l'anima alla spiritualità.

Il monito sembra risuonare nell'introduzione alla Muse chrestienne ou Recueil de poesies chrestiennes tirées des principaux poetes françoys, in cui si ribadisce:

elles ne sont qu'invocations de la misericorde et grace de Dieu pour estre delivré des concupiscences de la chair, et exciter son ame, aux choses spirituelles ${ }^{102}$

La silloge, pubblicata a Parigi nel 1582, è la raccolta di alcuni componimenti genericamente ispirati a valori cristiani, presenti nella poesia contemporanea, testimonianza dell'impegno spirituale di una lirica ronsardisante che la cultura protestante condanna in quanto mitologica, sensuale, profondamente laica ${ }^{103}$. Indirettamente

(97) Malipero Hieronimo, Petrarca spirituale, Venezia, Marcolino, 1536, in- $4^{\circ}$. Le citazioni successive sono tratte dall'esemplare della Biblioteca di Brera (AB. XVI. 52).

(98) Il volume esce due anni dopo in $-8^{\circ}$, cfr. S. CASALI, Gli annali della tipografia veneziana di Francesco Marcolini, (Forlì, 1861), Bologna, Fiammenghi, 1953, pp. 21-26, 78-79. Le altre edizioni sono del 1545, 1567, 1575, 1581, 1587.

(99) È lo stesso Petrarca ad invocare le giuste correzioni in un dialogo immaginario con Malipiero, posto ad introduzione del Canzoniere stesso, cfr. Malipero Hieronimo, Petrarca spirituale, Venezia, Marcolino, 1536, «Dialogo di Frate Hieronymo Maupetro Venetiano del Sacro ordine de minori di osservanza al Petrarcha Theologo et Spirituale introduttivo», ff. A-Bivr ${ }^{\circ}$

(100) A. Quondam, Riscrittura-Citazione-Parodia del codice. Il «Petrarca spirituale» di Girolamo Malipiero, (1978), in Il naso di Laura. Lingua e poesia lirica nella tradizione del Classicismo, Modena, Panini, 1991, pp. 203-262; U. Rozzo, L'espurgazione dei testi letterari nell'Italia del secondo cinquecento, in La censura libraria nell'Europa del secolo XVI, a cura di U. Rozzo, Udine, Forum, 1997, pp. 219271.
(101) Cfr. ff. z-BBr ${ }^{\circ}$. Il Petrarca spirituale è inserito integralmente anche nei due volumi di Rime spirituali, parte nuovamente raccolta da più autori, parte non più data in luce, Venezia, Al segno della Sapienza, 1550. La Canzone alla Vergine è nel secondo volume.

(102) Muse chrestienne ou Recueil de poesies chrestiennes tirées des principaux poëtes françoys, Paris, G. Malot, 1582, f. $228 \mathrm{v}^{\circ}$.

(103) Delle Rime del Petrarca la cultura protestante ammira proprio quei sonetti antiavignonesi che né Vellutello né Philieul hanno pubblicato. Nell'edizione dell'Opera omnia di Petrarca, stampata a Basilea, nel 1554 da Heinrich Petri, nel IV volume si trovano $\mathrm{i}$ sonetti censurati, cc. $12 \mathrm{r}^{\circ}$ e $15 \mathrm{r}^{\circ}$. Essi sono riproposti da Pier Paolo VergeRIO in Stanze del Berna con tre sonetti del Petrarca dove si parla dell'evangelio e della Corte di Roma, Tübingen, Morhard, 1557; Id. Alcuni importanti luochi tradotti fuor de le epistole latine di M. Francesco Petrarca [...] con tre suoi sonetti, Tübingen, Morhard, 1557. La curia ha condannato l'opera vergeriana negli Indici del 1559 e del 1564. Cfr. U. Rozzo, L'espurgazione dei testi letterari nell'Italia del Secondo Cinquecento, in La censura libraria nell'Europa del secolo ... cit. 
questa Musa cristiana, polemicamente cattolica, risente dell'influenza petrarchesca, il Petrarca del Trionfo della Morte, più che il poeta del Canzoniere; tuttavia, risuona un lessico penitenziale che alcuni fragmenta hanno squisitamente codificato ${ }^{104}$. Pochi i riferimenti a Maria che imitino direttamente la Canzone 366. Si ammira une «Estoille enfanter un Soleil, une Vierge accoucher $»^{105}$, una luce rassicurante che risplende nei versi petrarcheschi di Molza, Parabosco, Annibal Caro, Tansillo, Pagani ${ }^{106}$, imitati da Desportes, Bertaut, Du Perron; la Vergine del Petrarca, che salva dal tempestoso mare e conduce al porto di Cristo ${ }^{107}$, splendente $« E t$ comme un vestement le Soleil l'environne» ${ }^{108}$, surge nella poesia devozionale contemporanea per chinarsi dolorosamente in nuovi atteggiamenti: sofferente e lacrimante questa Madonna sembra destinata a perdere il tratto divino e mortale che l'equilibrio formale del Petrarca le aveva garantito. All'insegna di una cultura controriformata, infatti, il rinnovato lirismo mariano della seconda metà del Cinquecento esalta in Maria la figura della madre afflitta, compatita spettatrice dell'orrore della Passione e Morte del Figlio. Il gesuita Michel Coyssard nelle Institutions della Confrérie de la très-beureuse Vierge Marie, attribuisce «un si grand avancemment es lettres» ${ }^{109}$ al culto della Vergine che lo stesso Henri III promuove, incoraggiando una convergenza tra la retorica umanistica e la devozione destinata a sedurre e a commuovere un largo pubblico. Du Peyrat, Le Digne, lo stesso Coyssard traducono lo Stabat mater ${ }^{110}$, dedicano intere raccolte alla Vergine dolente ${ }^{111}$, «esplorée», «Pasle, descolorée, escoulant de ses yeux / Des perles d'Oriant un fleuve precieux» ${ }^{112}$, ne ricordano il tratto materno ${ }^{113}$, patetico, commo-

(104) Si veda per esempio il sonetto 11 e 17 di Desportes e i sonetti 313 e 257 di Petrarca.

(105) J. BerTaut Les Euvres poetiques, dernière édition, Paris, Toussainct du Bray, 1620, «Cantique sur la naissance de nostre Seigneur», vv. 47-48. Jean Bertaut ha tradotto i trattati delle Vergini di S. Ambrogio, cfr. Les trois discours de Sainct Ambroise, intitulez des Vierges, à sa sour Marceline, ouvrage digne d'être toujours entre les mains des religieuses [...], Paris, veuve M. Patisson, 1604.

(106) Cfr. per esempio i versi del frate Antonio Pagani, Rime spirituali, Venezia, Zaltieri, 1570, «Il Trionfo del Redentore»: Canzone II, p. 3, vv. 19-21 «Colei, che sola al mondo, per sovrana / Gratia, d'alte virtuti Imperatrice / Piacque si a Dio, che se la fece sposa»; Capitolo, p. 12, vv. 47 «Madre d'un tal Figliuol, del Padre Figlia» e il «Cantique sur la naissance de nostre Seigneur» di Jean Bertaut.

(107) I. HaberT, Les trois livres des Météores, avecques autres ouvres poëtiques (Odes; Sonnets; Bergeries; (Euvres chrestiennes), Paris, J. Richer, 1585, «CEuvres chrestiennes, Sonnets Spirituels», XVII, f. 6r , vv. 9-14: «O Royne des hauts Cieux chaste et saincte pucelle / Qui jouis du repos de la vie eternelle / Mer de Jesus Christ (divin reparateur / Du mortel genre humain) escoute ma priere, / Tire de ceste mer mon ame prisonniere / Et la conduis au port de Christ mon redempteur».

(108) J. Davy Du Perron, Les Diverses CEuvres, Cantique de la Vierge, Paris, A. Estienne, 1622, II, pp. 17-20.

(109) Cit. in T. Cave, Devotional Poetry in France 1570-1613, Cambridge, Cambridge University Press, 1969, p. 13.

(110) Cfr. H. Vaganay, Un sonnet italien peu connu; quatre traductions du Stabat Mater au XVI siècle, «Revue des bibliothèques», 21, 1911, pp. 393-401. Vaganay riporta la traduzione di G. Du Peyrat, (Paraphrase de l'Hymne Stabat mater dei, pp. 394-395), quella di Pierre Dorì (da L'Image de Vertu, pp. 396-397), di Michel Coyssard (da Les Hymnes sacrez, pp. 398-99) e di Nicolas Le Digne (da Recueil des Premiers Euvres Chrestiennes, pp. 400-401)

(111) Cfr. P. Doré, L'Image de Vertu, demonstrant la perfection et saincte vie de la bien beureuse Vierge Marie, Paris, N. Bonfons, 1549; J. DE BILly, Sonnets spirituels recueillis pour la plus art des anciens theologiens tant grecs que latins, Paris, N. Chesneau, 1573; G. Du Peyrat, Hymne de la Trinité, avec quelques sonnets spirituels, Paris, Linoncier, 1587; P. TAMisiER, Cantiques, bymnes, et prieres des saincts Peres [...], Lyon, B. Rigaud, 1590; F. Coster, Cinquante meditations de la vie et louange de la Vierge Marie. Avec sept Meditations sur le Cantique Salve Regina, Anverse, J. Mourentorf, 1590; M. Coyssard, Les Hymnes sacrez et odes spirituelles [...], Lyon, Jean Pillehote, 1594; A. FAvRE, Centurie premiere de sonnets spirituels de l'amour divin et de la penitence, Chambéry, Claude Pomar, 1595; N. Le Digne, Recueil des Premieres Euvres Chrestiennes, Paris, 1600.

(112) César De Nostredame, La Maria dolente. Au sieur Delsherms advocat tolosain, cit. in T. CAVE, Devotional Poetry in France, ... cit., «Appendix», p. 326: «Mais la vierge esplorée, innocente et sacrée, / Qui ceste brebis sanglante et massacrée / Treuver tant agreable et soüefve la mort / Sur le lict de la Croix qu'on diroit qu'elle dort, / Dessus ses pieds tramblans pres de la Croix dressée, / Du glaive de douleur d'outre en outre persée, / Pasle, descolorée, escoulant de ses yeux / Des perles d'Oriant, un fleuve precieux».

(113) J. Davy Du Perron, Les Diverses Euvres, Cantique de la Vierge, Paris, A. Estienne, 1622, II, p. 17: «Elle luy va monstrant pour flechir sa rigueur, / Les mammelles qui tendre au berceau l'allaitterent, / Dont le doux souvenir luy penetre 
vente. La «desolée mere» ${ }^{114}$, la «tres-dolente Dame» ${ }^{115}$ dei Vers chrestiens di Gabrielle de Coignard, che arriva ad immedesimarsi in Maria «seulette, sans espoux, / Vefve de ton enfant si gracieux et doux» ${ }^{116}$ torna nella Madonna di Anne de Marquets,

A quoy compararay-je, ô Vierge de Sion,

Ton angoisseux ennuy, ta douleur vehemente,

Sinon à l'ocean que la bize tourmente,

Qui ne se voit jamais sans agitation

Ceci se peut bien dire en contemplation

Du martyre cruel que la Vierge dolente

Ressentoit en son cœur, quand par mort violente

son Fils voulut payer nostre redemption:

Car son angoisse alors, a nulle autre seconde ${ }^{117}$

e preannuncia le tinte intensamente patetiche della Vergine in pianto di Jean de La Ceppède $^{118}$ et François de Malherbe ${ }^{119}$. Sembra inanzitutto si voglia mostrare questa Grande Madre dolente, che giganteggia, per esempio, nel frontespizio del terzo libro dei Théoremes ${ }^{120}$, emblema di una poesia devozionale che evoca la partecipazione emotiva del suo pubblico.

Perché la Vergine di Petrarca torni ad essere un modello privilegiato occorre rivolgersi nuovamente a una cultura di inedite confluenze: in una terra ricca di tradizioni, fra Mediovo e Rinascimento, tra Italia e Francia, sotto il segno della varietà e del dialogo tra sacro e profano, l'ultimo componimento del Canzoniere riconquista il valore di un fragmentum di armonia. Si tratta della cultura normanna, profondamente segnata dal culto mariano esaltato dai Puys del Palinod, da Caen a Rouen, attraversata,

le cœur, / Et les flancs bien-heureux qui neuf mois le porterent».

(114) G. De Coignard Euvres Chretiennes, éd critique par Colette H. Winn, Discours sur la passion de nostre sauveur Jesus-Christ, vv. 794-801: «Ainsi que celle-là ta desolée mere, / Laquelle s'avançoit pour recognoistre, helas, / Son enfant bien-aymé qu'on menoit au trespas. / Ha! vierge, je te pry' de te tirer arriere, / Et ne regarde point la cruelle baniere / Que l'on faisoit porter à ton fils bien aymé, / Qui transperçoit ton cœur d'un glaive envenimé».

(115) Ibidem, vv. 817-822: «Et voyant ses beaux yeux de douleurs obscurcis, / Regardant ces travaux la tres-dolente Dame, / Un glaive de douleur lors transperça son ame, / Et dix mille cousteaux luy ouvrirent le cœur, / Mouillant son sacré corps d'une froide sueur, / Les yeux luy ternissoient d'angoisseuse tristesse, / Et ses membres sacrez deffailloyent de foiblesse».

(116) Ibidem, «Complainte de la vierge Marie», vv. 3-4. Nell'Introduction Colette H. WINN sottolinea «c'est surtout comme la "créature charnelle" que Dieu par sa bonté a placée au centre des événements miraculeux et, tout spécialement, comme la mère de Jésus Christ que Gabrielle Coignard se plaît à glorifier Marie [...]. C'est dans la chair que la mère participe à l'horreur des derniers instants. Gabrielle Coignard s'appesantit sur la compassion viscérale de la Vierge», pp. 86-87.

(117) Anne DE MARQuets, Sonnets spirituels, éd. critique par Gary Ferguson, Genève, Droz, 1997, «Pour la feste de nostre dame de Pitié», CCCXCVII, p. 321. La poesia devozionale della re- ligiosa domenicana risulta comunque più sobria e discreta dei suoi contemporanei, comme sottolinea Ferguson, «... on constate dans ses vers comme une sorte de retenue dans l'excès et l'exagération. [...] on a affaire à un baroque tout jeune, un baroque dont la fogue naissante est déjà modérée par sa soumission à l'esthétique et à la discipline monastiques», Introduction, p. 56. Cfr. anche E. BERRIOTSAlvadore, Les Femmes dans la société française de la Renaissance, Genève, Droz, 1990, pp. 296-299.

(118) J. De la Ceppede, Les Théorèmes sur le sacré mystère de nostre rédemption, éd critique par Y. Quenot, II voll., Paris, 1988 e 1989. Cfr. Y. QueNOT, La Vierge dans les Théorèmes de La Ceppède, «XVII ${ }^{e}$ Siècle», 170, 1991, pp. 27-38.

(119) Cfr. A. Muller, La Poésie religieuse catholique de Marot à Malherbe, Paris, R. de Foulon, 1950; T. Cave, Devotional Poetry in France 15701613, Cambridge, Cambridge University Press, 1969. Cfr. anche M. Jeanneret, Poésie et tradition biblique au XVIe siècle. Recherches stylistiques sur les paraphrases des psaumes de Marot à Malherbe, Paris, Corti, 1969, II parte, cap. IV, La poésie pénitentielle; cap. V, La littérature pénitentielle.

(120) L. C. BLACK, Mary or Urania: a study of the invocation to Book. III of La Ceppède's Théorèmes, «Explorations in Renaissance Culture», 8-9, 1982-83, pp. 33-44; J. PlantiÉ, J. Yeremian, Un "théorème" de plus: le frontispice de Théorèmes de Jean de La Ceppède, «XVII ${ }^{\mathrm{e}}$ Siècle», 143, 1984, pp. 89-105; L. C. BLACK, Iconography of the devotional aesthetics: a study of the title page to La Ceppède's Théorèmes, «Explorations in Renaissance Culture», 15, 1989, pp. 59-82. 
al tempo stesso, da una forte rinascita spirituale ${ }^{121}$ che sposa all'interesse per la cabala, la conoscenza della Bibbia, l'amore per letteratura italiana del XIV e XV secolo. Le savant orientaliste normanno Guy Le Fèvre de la Boderie, infatti, eminente membro dell'Académie du Palais di Henri III, è uno dei protagonisti di questa felice stagione dello spirito e guarda all'Italia, - a Iacopone da Todi, a Caterina da Siena, ma anche a Dante, Petrarca e Sannazzaro ${ }^{122}$ - per rinvigorire l'appello alla rigenerazione cui è destinata la cultura gallica. L'anelito ad un'egemonia dello spirito che si armonizza con la restauratio di una grande monarchia universale ${ }^{123}$, si volge nuovamente aux ancêtres italiens e attinge luminosità e purezza alle fonti più chiare dell'antica Etruria: è in questa atmosfera che la Canzone alla Vergine ritrova un inedito smalto, tradotta da Le Fèvre e pubblicata, nel $1578^{124}$, insieme alla versione francese del XXXIII canto del Paradiso ${ }^{125}$ di Dante. Poeta del Puys de l'Immaculée Conception, «disciple élu d'un autre normand qui se considérait le fils de la Vierge» ${ }^{126}$, quale Guillaume Postel, Guy Le Fèvre de la Boderie ricorre costantemente in tutta la sua opera alla figura della Vergine $^{127}$, donna di intera purezza, imago dei, che riflette armoniosomente la sacra e sublime unità delle origini. Senza approfondire la complessità di questa "teosofia virginale", che la critica ha recentemente messo in luce ${ }^{128}$, ci interessa riconoscere in quest'ultima versione della Canzone petrarchesca una rinnovata forma di dignitas hominis che, ben lungi dal compiacere il gusto controriformato della Passione cui assiste dolente Maria, torna a compiersi nella teologia dell'Incarnazione e rende la Vergine il luminoso modello di una «déification de l'homme» ${ }^{129}$.

Affascinato dalla riscrittura del Petrarca che concilia gli studia divinitatis alle bumanae litterae, Le Fèvre riprende accuratamente i riferimenti alla Bibbia di cui è costellata l'intera Canzone ${ }^{130}$, e l'intarsio di rimandi ai Padri della Chiesa, le limpide

(121) «restauration religieuse normande, baptisée faute de mieux Contre-Réforme en dépit de ses couleurs gallicanes, et point toujours orthodoxes», J.-F. Maillard, Postel et ses disciples normands, in Guillaume Postel, Paris, Ed. de la Maisinie-Guy Trédaniel, 1985, p. 85.

(122) Cfr. R. GorRIs, Blaise de Vigenère et Guy Le Fère de La Boderie traducteurs de l'italien in Blaise de Vigenère, poète et mythographe au temps de Henri III, "Cahiers V. L. Saulnier", 11, Paris, Pens, 1994 , pp. 77-100 ora in Alla corte del principe, Traduzione, romanzo, alchimia scienza e politica tra Italia e Francia nel Rinascimento, Ferrara, Università degli Studi di Ferrara, 1996, pp. 71-94; J.-F. MaIllard, A l'ombre de Sannazar et de Georges de Venise: textes oubliés de Guy Le Fèvre de La Boderie in Poésie encyclopédique et Kabbale chrétienne. Onze études sur Guy Le Fèvre de La Boderie, Paris, Champion, 1999 pp. 223-245.

(123) M. Fumaroli, Aulae Arcana. Rbétorique et politique à la cour de France sous Henry III et Henri IV, «Le Journal des Savants», 1981, pp. 137-189.

(124) Hymne à la Vierge sacrée, du Toscan Petrarque. A Monsieur l'Evesque du Puiz Chancellier de la Royne mere des Roys, in Hymnes ecclesiastiques, cantiques spirituelz et autres Meslanges poetiques au tres chrestien Henri IIIe, roi de France et de Pologne, par Guy Le Fevre de La Boderie, Secrettaire de Monseigneur, frere unique du roi et son interprete aux langues estrangeres, Paris, Robert Le Mangnier, 1578 , ff. $227 \mathrm{r}^{\circ}-230 \mathrm{r}^{\circ}$.

(125) A la Vierge mere de Dieu. De Dante Poëte Toscan, in Hymnes ecclesiastiques, ... cit., ff. $167 \mathrm{r}^{\circ}$ $169 v^{\circ}$. Su questa traduzione cfr. in particolare P. DE NolHac, Une traduction de Dante au temps de la
Pléiade. Guy Le Fèvre de La Boderie, «Bulletin du $\mathrm{VI}^{\mathrm{e}}$ centenaire de la mort de Dante», janvier 1922, pp. 528-530.

(126) R. GORRIS Traduire la Vierge. Guy Le Fèvre de La Boderie poète et traducteur de Dante, de Pétrarque et du "De Partu Virginis" de Jacobo Sannazzaro, in Poésie encyclopédique et Kabbale chrétienne. Onze études sur Guy Le Fèvre de La Boderie, réunis par F. Roudaut, Paris, Champion, ora in Alla corte del principe. Traduzione, romanzo, alchimia scienza e politica tra Itlia e Francia nel Rinascimento, Ferrara, Università degli Studi di Ferrara, 1996, p. 147.

(127) Le Fèvre traduce anche il De Partu Virginis di Jacopo Sannazzaro, cfr. De l'enfantement de la Vierge Royne des Vierges, imité du latin de Jacques Sannazar, Gentilhomme napolitain, par Guy Le Fèvre de la Boderie, Paris, Abel l'Angelier, 1582. Sulle sue composizioni alla Vergine e su queste traduzioni cf. R. GoRRIS, Traduire la Vierge..., cit. pp. $141-170$

(128) Cfr. in particolare Poésie encyclopédique et Kabbale chrétienne. Onze études sur Guy Le Fèvre ... cit. e F. Roudaut, Le point centrique. Contribution à l'étude de Guy Le Fèvre de la Boderie (15411598), Paris, Klincksieck, 1992.

(129) Cfr. J.-F. Maillard, A l'ombre de Sannazar et de Georges de Venise: textes oubliés de Guy Le Fèvre de La Boderie in Poésie encyclopédique et Kabbale ... cit., p. 227.

(130) Cfr., per esempio, la traduzione del riferimento alla parabola delle vergini sagge, «O Vierge sage, et du beau nombre l'une, / De celles là qui portent en leurs cueurs / La lampe ardente...» vv. 19-21. 
sources della fede ${ }^{131}$, quali S.Agostino, «Catholicae Ecclesiae clarissima lumina» ${ }^{132}$. Il prezioso materiale si arricchisce dei temi più tradizionali della poesia palinodique $e^{133}$, cui si affiancano armoniosamente gli epiteti lauriani, le rime dafnee che il Petrarca ha già rivestito di intensità e luce ${ }^{134}$. Pur sostanzialmente fedele all'originale, Le Fèvre accentua tale luminosità, dilata su più piani la misura che definisce la Canzone del Petrarca, a vantaggio di una sistematica amplificatio, la cifra più significativa della sua traduzione ${ }^{135}$. I versi consacrati alla gloria di Maria si raddoppiano in un gioco di ridondanze che riecheggia il peana, il canto di esaltazione più che la litania, secondo una tradizione già evocata da Beroaldo e Bade.

Vergine gloriosa, $\quad$ O toy Vierge d'honneur

donna del Re, che nostri lacci ha sciolti, De triomphe et bonheur

(vv. 48-49)

Du Roy des Roys la Dame non jalouse

Qui a noz lacs rompus et deliez (vv. 66-60)

s'a' Tuoi preghi, o Maria,

Si par ton oraison

Vergine dolce e pia,

ove'l fallo abondò, la gratia abonda

(vv. 60-62)

Vierge en toute saison,

Sur tout Vierge en clarté radieuse,

Douce, piteuse, et humaine aux humains,

La grace abonde où abonda la faute

(vv. 83-87)

La stella diventa «Astre doré», «chiara lampa» che il Petrarca rendeva efficacemente visibile, e che Le Fèvre traduce in un'intensa visione:

chiara lampa

(v. 16)
La lampe ardente, aincois qui as la Lune

Dessouz tes pieds, et les sainctes liqueurs Qui font flamber ta lampe la plus belle (v. 21-23)

L'amplificatio dilata anche lo spazio concreto che Maria illumina fra terra e cie$\mathrm{lo}^{136}$, e traduce in un'iperbole quel movimento ascensionale che attraversa la Canzone del Petrarca:

salisti al Ciel, onde i miei preghi ascolti (v. 42)
Au Ciel des Cieux as esté envolée Dont tu entends ce mien hymne dictée (vv. 56-58)
(131) La quarta sessione del Concilio di Trento (1546) ribadisce l'importanza della testimonianza dei Padri della Chiesa come fonte primaria dell'apologetica cattolica. Cfr. J. Delumeau, Le Catholicisme entre Luther et Voltaire, Paris, Puf, 1979 , p. 54.

(132) G. Le FÈvre de La Boderie, Novum Jeesu Christi Testamentum, complectens, praeter Vulgatam, Guidonis Fabricii e Syriaco et Benedicti Ariae Montani translationes, Anvers, Christ. Plantin, $1571, \mathrm{f} .+\mathrm{vr}^{\circ}$.

(133) Cfr. il «rond et beau temple » (v. 75), l'«Emperiere» (v. 137 e v. 170), la «lampe ardente» (v. 21), «la fontaine» (v. 59), il «cloistre» (v. 108), il «brigantin» del congedo. Vedi G. Gros, Le Poète, la Vierge et le Prince du Puy, Paris, Klincksieck, 1992, pp. 182-196, Incipit et ligne palinodiale, dei diversi componimenti.; G. GROS, Le rondeau marial au Puy de Rouen, «Nouvelle revue du XVI ${ }^{\mathrm{e}}$ siècle», 14 / 2, pp. 117-154.

(134) Tornano anche le figure di Eva e Medusa: «le deuil d'Eve» (v. 50); «Meduse et mon peché» (v. 153).

(135) Cfr. anche il riferimento al naufragio mortale:

et ò già da vicin l'ultime strida

Je ia sentant la vague sur ma teste

(v. 71)

Et de la Mort l'horrible espouvantail (vv. 97-98)

(136) cui né prima fu simil né seconda, (v. 55)

Qui n'euz jamais en ce rond et beau temple Tout rayonnant de splendeurs et clartez Nulle premiere, de pareille ou seconde (vv. 75-77) 
L'«hymne», ribadisce foneticamente la presenza del possessivo «mien» già privilegiato dall'accento del verso, segno di un'invadenza del soggetto, che è una delle conquiste della poesia petrarchesca: Le Fèvre si riconosce al centro dell'invocazione $^{137}$ e si immedesima negli affanni evocati dal Petrarca, alludendo a un travaglio personale, a una peregrinazione, anche metaforica, nelle diverse e complesse strade della conoscenza ${ }^{138}$. Il gioco di distanza e ravvicinamento torna a concretizzarsi nella figura di Maria, «Du grand Soleil la grace tu as euë»(v. 3), «Qui ceste vie illustres de lumière, / Et vas ornant l'autre plus claire qu'or» ${ }^{139}$, e trascende nell'uomo stesso: la creatura - dotata di ragione - deve colmare uno scarto per recuperare quell'originaria scintilla divina che gli ha dato la vita.

ben ch'i sia terra, e Tu del ciel regina (v. 13)

non guardar me, ma chi degnò crearme; no' l mio valor, ma l'alta Sua sembianza ch'è in me, Ti muova a curar d'uom si basso. (vv. 108-110) bienque ne soye (hormise la raison) Rien sinon terre, et toi du Ciel la Reyne (vv. 17-18)

En moy indigne, ains le Createur digne Qui a daigné me former par compas Non ma valeur, mais l'Image divine (vv. 149-151)

L'allusione al compasso evoca una perfezione concentrica che riunisce in un unico spazio purificante anche l'esperienza umana ${ }^{140}$ e l'espressione di sé che l'uomo sa tradurre in arte:

Je sacre desormais / Et purge pour jamais / Non le seul corps qui n'est que terre et poudre, / Mais à ton nom l'Ame, l'Amour, la main, / Le cueur, la langue, et les soupirs et larmes (vv. 174-177)

L'uomo-poeta-profeta sembra volersi librare alle altezza di Colei che «humaine des humains...Mere, fille et Espouse» risiede radiosa «En l'Eternité stable»: la conversione, allora, acquista i contorni di un ritorno all'effigie divina che ha plasmato l'umano, una riconciliazione con Sé più che un pentimento destinato a rinnegare il passato. Le Fèvre de la Boderie tralascia il «cor contrito et humile», il «miserere», ma esalta con vigore l'unità, il Principio di Bene che fonda l'universo tutto e si impone nella sua versione:

(137) per Te il tuo Figliuol, et quel del sommo Padre

o fenestra del ciel lucente altera, venne a salvarne in su li extremi gironi (vv. 30-32)

ne le cui sante piaghe

prego ch'appaghe il cor, vera beatrice (vv. 51-52)

Veuille par toy le Filz du souverain Pere Et ton filz cher, dessus le derniers jours Pour me sauver se presenter prospere, Si qu'apres mort je vive à luy toujours O fenestre des Cieux (vv. 41-45) Et son costé,

mon cueur se purge immonde (vv. 71-72).

(138) non è stata la mia vita altro ch'affanno Mortal bellezza, atti, et parole m'anno, tutta ingombrata l'alma.

Rien hors d'ennuy je n'ay repeu ma vie,
Errant et voyager

En pays estranger

En maint terroir maincte route ay suivie (vv. 119-122)

(139) vv 39-40, cfr. la Canzone del Petrarca, v. 29, «ch'allumi questa vita e l'altra adorni».

(140) Le Fèvre traduce letteralmente la strofa con cui Petrarca dà impressionante rilievo anche all'umano peccare:

Ricordati che fece il peccar nostro

prender Dio, per scamparne,

humana carne, al tuo virginal chiostro

(vv. 76-78)

Souvienne-toy que noz pechez ont faict

Prendre au grand Dieu de tout le monde maistre / Pour nous purger de vice ord et infect / Humain cher en ton virginal cloistre. (vv. 105-108) 
del comun principio amor T'induca miserere d'un cor contrito, humile;
Le grand amour du Principe commun Te veuille induire à m'estre bonne amye Ayant pitié d'un cueur qui vit en L'UN. (vv. 164-166)

Il traduttore, kabbaliste chrétien, infonde nei suoi versi uno spirito di concordia filosofica e teosofica che dilata e trasforma, in parte, i contenuti dell'originale petrarchesco; tuttavia, in tale rielaborazione la Canzone alla Vergine torna a brillare di luce propria, fragmentum in sé concluso, al quale Le Fèvre riconosce una sostanza sincretica esemplare: armonioso mosaico di generi, tradizioni, tematiche eterogenee quanto convergenti, il poema dà forma alla Riconciliazione di cui la Vergine è segno per eccellenza. Ne è testimonianza privilegiata, ancora una volta, la strofa conclusiva:

Le jour se haste, et la nuict me talonne,

Et le temps bref et court

Plustost s'envole et court

Qu'un brigantin que les ondes sillonne,

Qu'un dard, ou qu'un eclair,

Vierge unique et sans pair,

La conscience a poing mon cueur impur,

Mais or la mort plus le poingt et le presse,

Fais que ton Filz vray homme et vray Dieu pur

Reçoive en paix mon Esprit qui me laisse (vv. 181-190)

Le Fèvre riprende il modulo binario scandito dal ripetersi di congiunzioni, di coppie di qualificativi sinonimici e verbi semanticamente affini e intensifica il ritmo dell'originale con l'omofonia dell'aggettivo-verbo «court». L'amplificatio, «Qu'un brigantin...éclair», alimenta il gioco di parallelismi evocando un mare e un cielo perfettamente contigui e attraversati dallo stesso movimento rapido che congiunge l'acqua, il fuoco, l'aria e la luce. La lunga sequenza di possessivi (me, mon, mon, me) impone la presenza di un soggetto destinato a purificarsi attraverso il Figlio-uomo che è - iperbole di perfezione - Dieu pur.

Ben lungi dall'essere l'ultimo tassello di un percorso tracciato nelle Rime, la Canzone alla Vergine se ne distingue, alta espressione di un'armonia di forme e contenuti che mantengono nei secoli il valore di un'iniziazione. Agli occhi di questo orientalista appassionato di cabala e di neoplatonismo, inedito protagonista della rinascita cattolica promossa da Henri III, il diario sentimentale del poeta toscano ha perso ogni interesse, diluito in infinite imitazioni, ridotto a vuota maniera. A proposito del petrarchismo amoroso conosciuto in gioventù, Le Fèvre ammonisce nell'Encyclie, «Quittons du fol amour les feintes courtoisies» ${ }^{141}$. Familier dei grandi umanisti del primo Cinquecento e apologeta di quella dignitas bominis che essi hanno ritrovato innanzitutto nei Padri della Chiesa ${ }^{142}$, Le Fèvre torna a privilegiare con la stessa profonda ammirazione l'ultimo componimento del Canzoniere, un prisma dai bagliori divini

(141) G. Le FÈvre de La BoderIE, L'Encyclie des Secrets de l'Eternité. A tres hault et Tres-Illustre Prince Monseigneur le duc d'Allençon frere du Roy tres-chrestien Charles Neufième, Anvers, imprimerie de Christofle Plantin, 1571, p. 248.

(142) Sulle fonti patristiche dell'opera di Le Fèvre, cfr. G. LE FÈvre de La Boderie, La Galliade, éd. critique par F. Roudaut, Paris, Klincksieck,
1993, Introduction, pp. 50-54; G. LE FÈvRE de LA Boderie, Diverses meslanges poétiques, éd. critique par R. Gorris, Genève, Droz, 1993. Sul concetto di dignitas hominis nell'Umanesimo e nel Rinascimento cfr. L. Sozzi, "Un désir ardent". Etudes sur la «dignité de l'bomme»à la Renaissance, Torino, Il Segnalibro, 1997. 
e dalle molteplici forme perfette che, per opposizione, rivela la decadenza delle rime galanti laurane e, implicitamente, della cultura italiana che le ha rese fatuo modello.

Tout ainsi les deux Seurs errant 's par la Toscane

Ont acquis non un teint et couleur de basane,

Ainçois un teint fardé, un maintient decevant,

Et gestes plus lascifs qu'oncques auparavant,

Un Amour feint qui sçait le transy contrefaire,

Bref l'air Italien qui à tous ne peut plaire:

Et des noouveaux attours, ou plustost refripez

Sur les Latins et Grecs subtilement gripez,

Si que chacune Nymfe, autrefois simple et sainte,

Fut lors de plus en plus Italienne et feinte ${ }^{143}$

Nella scelta della Canzone alla Vergine Guy Le Fèvre de la Boderie predilige, all'ereditarietà geografica del Canonico di Carpentras, un'affinità elettiva ben più profonda ma afferma, nell'ammirare quest'unico fragmentum delle Rime sparse, la decadenza delle litterae d'Italia, ovvero, ancora una volta, l'assoluta superiorità della cultura "gallica" contemporanea:

Tout ainsi les deux Seurs en leurs derniers ans vieux

Apres avoir erré des Bardes aux Hebrieux,[...]

Des Gregeois aux Latins, et des Latins nerveux

Aux modernes Toscans leurs fils et leurs neveux [...]

Repasserent les monts, et revindrent en France

Que lon nommoit encor la Gaule en leur enfance ${ }^{144}$

ALESSANDRA PREDA

(143) G. Le Fèvre de la Boderie, La Galliade, éd. critique par F. Roudaut, Paris, Klincksieck, 1993, p. 547, Cercle V, vv. 889-9898.
(144) Ibidem, pp. 547-548, Cercle V, vv. 915-16; 919-20; 929-30. 